\title{
Analogues of gravity-induced instabilities in anisotropic metamaterials
}

\author{
Caio C. Holanda Ribeiro $\odot^{*}$ and Daniel A. Turolla Vanzella $\odot^{\dagger}$ \\ Instituto de Física de São Carlos, Universidade de São Paulo, Caixa Postal 369, 13560-970, São Carlos, São Paulo, Brazil
}

(Received 27 November 2019; accepted 11 February 2020; published 9 March 2020)

\begin{abstract}
In the context of field theory in curved space-times, it is known that suitable background space-time geometries can trigger instabilities of fields, leading to exponential growth of their (quantum and classical) fluctuations, a phenomenon called vacuum awakening in the quantum context, which in some classical scenarios seeds spontaneous scalarization or vectorization. Despite its conceptual interest, an actual observation in nature of this effect is uncertain since it depends on the existence of fields with appropriate masses and couplings in strong-gravity regimes. Here, we propose analogues for this gravity-induced instability based on nonlinear optics of metamaterials which could, in principle, be observed in laboratory.
\end{abstract}

DOI: 10.1103/PhysRevResearch.2.013281

\section{INTRODUCTION}

The influence of a background material medium on the propagation of mechanic and electromagnetic waves is well known to be formally analogous to that of an effective curved space-time geometry. This idea was first presented, in the electromagnetic context, by Gordon in 1923 [1] and it has since been developed in a number of different scenarios, particularly after Unruh's [2] and Visser's [3] works on acoustic analogues of black holes and their associated Hawking-type radiation. More recent applications of this formal analogy include mimicking in material media quantum light-cone fluctuations [4] and anisotropy in cosmological space-times [5]. The most appealing feature of these condensed-matter analogues of gravitational backgrounds is the possibility of observing in laboratory subtle but conceptually interesting effects which can be virtually unobservable in their original contexts, Hawking radiation being certainly the most emblematic among them, with claims of having already been observed in laboratory [6-8].

An interesting effect in the context of (quantum) fields in curved space-times is the triggering of field instabilities due to the background space-time geometry, a phenomenon called vacuum awakening in the quantum context [9-12]. These gravity-induced instabilities exponentially amplify vacuum fluctuations to the point they decohere and seed classical perturbations [13], which, depending on field parameters, eventually evolve to a nonzero classical field configuration ("spontaneous scalarization" in the case of scalar fields [14-17]), stabilizing the whole system. More recently, this mechanism was also predicted to occur for massless

\footnotetext{
*caiocesarribeiro@ifsc.usp.br

†vanzella@ifsc.usp.br
}

Published by the American Physical Society under the terms of the Creative Commons Attribution 4.0 International license. Further distribution of this work must maintain attribution to the author(s) and the published article's title, journal citation, and DOI. spin-1 fields through appropriate nonminimal couplings [18] and, in analogy with the scalar case, the stabilization process was termed "spontaneous vectorization." To the best of our knowledge, condensed-matter and optical analogues of these gravity-induced instabilities have not been proposed to this date. In this work, we propose and explore possible analogues of gravity-induced instabilities in the context of electromagnetism in polarizable and magnetizable anisotropic (meta)materials.

Electromagnetic instabilities in flat space-time are expected to occur in some materials. One celebrated example appeared in the context of plasma physics in the late 1950s and became known as Weibel instability [19]. The system, a neutral plasma whose components have anisotropic velocity distribution, possesses growing electromagnetic transverse waves. Related effects have been studied since then, with recent applications to solar plasma instability [20] and solidstate devices [21]. Moreover, causal aspects of classical propagation in active materials were discussed in Ref. [22], where properties of the refractive index were established. Nevertheless, aside from the fairly recurrence in the literature, usually quantization in such scenarios is not considered [23-25] or it is regarded as inconsistent [26,27].

It is noteworthy that instability of the electromagnetic field is always accompanied by evolution of the background, ending with the stabilization of the system as a whole. In the case of gravity-induced instability, the gravitational field changes with time, whereas electromagnetic instability in the presence of plasmas involves growing plasmons. In the case of electromagnetic fields in the presence of matter, for whatever form of the interaction with the background, the field's evolution is ruled by Maxwell's equations in the presence of polarizable and magnetizable media, and the interaction with the background is encapsulated in the functional dependence of the electric displacement (magnetic) vector field D $(\mathbf{H})$ with the true (microscopic) fields $\mathbf{E}$ and $\mathbf{B}$. If the magnitudes involved are small (e.g., in the beginning of the instability action), these functional relations become linear and one may find the form of the coefficients for such systems. For the case of Weibel instability, for instance, if the velocity anisotropy is 
taken in the $z$ direction, the instability is modeled by a negative squared refractive index in the direction perpendicular to $z$.

We apply Gordon's method to propose a family of opticalbased analogue models for electromagnetic fields presenting instabilities in curved space-times. We show how anisotropies of the background enter the effective equations in the form of nonminimal couplings, and in the case of strong anisotropy (just like for the Weibel instability), this coupling results in unstable solutions. We also discuss that for these systems the stabilization process occurs through the nonlinear nature of the background, which may seed spontaneous vectorization in analogy to the Einstein's field equations in the gravitational scenario.

The paper is organized as follows. In Sec. II, we present the covariant formalism of electromagnetism in anisotropic polarizable and magnetizable materials, establishing the formal analogy with nonminimally coupled electromagnetism in curved space-times. In Sec. II A, we consider a particular type of nonminimal coupling inspired by one-loop quantum electrodynamics (QED) corrections to electromagnetism in curved space-times. In Sec. III, we apply the formalism presented in the previous section to the scenario of a planesymmetric anisotropic medium at rest in an inertial frame. Although plane-symmetric curved space-times (in four dimensions) are not really (physically) appealing, we consider this scenario for its simplicity and for its possible implications for the physics of the material medium. We construct the electromagnetic quantum-field operator $\hat{\mathbf{A}}$ (in the generalized Coulomb gauge) in the standard-vacuum representation, discuss the conditions for appearance of instabilities and their types (Sec. III A), and present a concrete example (homogeneous medium; Sec. III B) where calculations can be carried over to the end. In Sec. IV, we repeat the treatment of the previous section, but now for a more appealing scenario on the gravitational side: spherically symmetric, stationary anisotropic media. Conditions for triggering instabilities and their types are shown to be very similar to those in the plane-symmetric case (Sec. IV A). As a concrete application, in Sec. IV B we show how to mimic QED-inspired nonminimally coupled electromagnetism in the background space-time of a Schwarzschild black hole. Then, Sec. V is dedicated to discuss possible stabilization mechanisms which might bear analogy to some curved-space-time phenomena, such as spontaneous vectorization [18] and particle bursts due to tachyonic instability [28]. Finally, in Sec. VI we present some final remarks. We leave for an Appendix tedious calculations related to the orthonormalization of modes of Sec. IV. We adopt the abstract-index notation to represent tensorial quantities (see, e.g., Ref. [29]) and, unless stated otherwise, we use natural units (in which $\hbar=c=1$ ).

\section{COVARIANT ELECTROMAGNETISM IN ANISOTROPIC MATERIAL MEDIA}

Electromagnetism in material media, in flat space-time and in the absence of free charges, is described by two antisymmetric (observer-independent) tensors, $F_{a b}$ and $G^{a b}$, satisfying the macroscopic covariant Maxwell's equations

$$
\begin{gathered}
\partial_{a} G^{a b}=0, \\
\partial_{[a} F_{b c]}=0,
\end{gathered}
$$

where $\partial_{a}$ is the derivative operator compatible with the flat metric $\eta_{a b}$ (but in arbitrary coordinates) and the square brackets denote antisymmetrization over the indices enclosed by them. These equations must be supplemented by mediumdependent constitutive relations between $F_{a b}$ and $G^{a b}$, as well as initial and boundary conditions, in order to provide a wellposed problem. These constitutive relations are usually set at the level of (observer-dependent) fields $E_{a}, B^{a}, D^{a}$, and $H_{a}$, related to $F_{a b}$ and $G^{a b}$ through

$$
\begin{aligned}
E_{a} & =F_{a b} u^{b}, \\
D^{a} & =G^{a b} u_{b}, \\
B^{a} & =-\frac{1}{2} \epsilon^{a b c d} F_{b c} u_{d}, \\
H_{a} & =-\frac{1}{2} \epsilon_{a b c d} G^{b c} u^{d},
\end{aligned}
$$

where $u^{a}$ is the four-velocity of the observer measuring these fields and $\epsilon_{a b c d}$ is the Levi-Civita pseudotensor [with $\epsilon_{0123}=$ $\left.\sqrt{-\eta}, \eta:=\operatorname{det}\left(\eta_{\mu \nu}\right)\right]$. Moreover, the constitutive relations usually take a simpler form in the reference frame in which the medium is (locally and instantaneously) at rest.

Here, we consider a polarizable and magnetizable medium whose constitutive relations in its instantaneous rest frame take the form

$$
\begin{aligned}
& D^{a}=\varepsilon^{a b} E_{b}, \\
& H_{a}=\mu_{a b} B^{b},
\end{aligned}
$$

where the tensors $\varepsilon^{a b}$ and $\mu_{a b}$ may depend on space-time coordinates, and the system is assumed dispersionless. We return to this point later. The fact that Eqs. (7) and (8) are valid in the medium's instantaneous rest frame means that the fields $E_{a}, B^{a}, D^{a}$, and $H_{a}$ appearing in them are related to $F_{a b}$ and $G^{a b}$ through Eqs. (3)-(6) with $u^{a}=v^{a}$, the medium's four-velocity field. We proceed by splitting the "spatial" [30] tensors $\varepsilon^{a b}$ and $\mu_{a b}$ into isotropic and traceless anisotropic parts,

$$
\begin{aligned}
\varepsilon^{a b} & =\varepsilon h^{a b}+\chi_{(\varepsilon)}^{a b}, \\
\mu_{a b} & =\mu^{-1} h_{a b}+\chi_{a b}^{(\mu)},
\end{aligned}
$$

where $h_{b}^{a}:=\delta_{b}^{a}+v^{a} v_{b}$ is the projection operator orthogonal to $v^{a}$. Inverting Eqs. (4) and (6) (with $u^{a}=v^{a}$ ),

$$
G^{a b}=2 v^{[a} D^{b]}-\epsilon^{a b c d} H_{c} v_{d},
$$

and substituting Eqs. (7)-(10) and (3) and (5), we obtain

$$
G^{a b}=\left(g^{a c} g^{b d}+\chi^{a b c d}\right) F_{c d},
$$

where we have defined the tensors

$$
\begin{aligned}
g^{a b}:= & \frac{1}{\sqrt{n}}\left[\eta^{a b}-\left(n^{2}-1\right) v^{a} v^{b}\right], \\
\chi^{a b c d}:= & \left(\frac{n}{\mu}-1\right) g^{a[c} g^{d] b}-2 \chi_{(\varepsilon)}^{[a \mid[c} v^{d]} v^{\mid b]} \\
& +\frac{1}{2} \epsilon^{a b e f} \epsilon^{c d g h} \chi_{e g}^{(\mu)} v_{f} v_{h},
\end{aligned}
$$

and the squared refractive index $n^{2}=\mu \varepsilon$. The idea, then, is to consider the symmetric tensor $g_{a b}$, defined through $g_{a b} g^{b c}=$ $\delta_{a}^{c}$, as an effective metric of a curved background space-time perceived by the electromagnetic field $F_{a b}$. Note that the 
components of $g^{a b}$ and $\eta^{a b}$ satisfy

$$
\operatorname{det}\left(g^{\alpha \beta}\right)=\operatorname{det}\left(\eta^{\alpha \beta}\right)
$$

and, thus, $\sqrt{-\eta}=\sqrt{-g}$, where $g:=\operatorname{det}\left(g_{\alpha \beta}\right)$. One can easily check that $g_{a b}$ is explicitly given by

$$
g_{a b}=\sqrt{n}\left[\eta_{a b}+\frac{\left(n^{2}-1\right)}{n^{2}} v_{a} v_{b}\right] .
$$

Therefore, in an arbitrary coordinate system, Eq. (1) reads as

$$
0=\frac{1}{\sqrt{-\eta}} \partial_{\alpha}\left(\sqrt{-\eta} G^{\alpha \beta}\right)=\frac{1}{\sqrt{-g}} \partial_{\alpha}\left(\sqrt{-g} G^{\alpha \beta}\right) .
$$

Up to this point, it was understood that the physical background metric $\eta_{a b}$ and its inverse $\eta^{a b}$ were responsible for lowering and raising tensorial indices. Now, with the introduction of an effective metric $g_{a b}$, we should be careful when performing these isomorphisms. In order to minimize chances of confusion, we shall avoid lowering and raising tensorial indices using the effective metric, making explicit most appearances of $g_{a b}$ and $g^{a b}$ in the equations below, with few exceptions which will be clearly stated. One obvious exception is the definition of $g_{a b}$ as the inverse of $g^{a b}$. Another such exception is the use of $\nabla_{a}$ to denote covariant derivative compatible with $g_{a b}$. With this in mind, from Eqs. (2) and (17), the electromagnetic tensor $F_{a b}$ satisfies

$$
\begin{aligned}
& 0=\nabla_{[a} F_{b c]}, \\
& 0=\nabla_{a}\left[\left(g^{a c} g^{b d}+\chi^{a b c d}\right) F_{c d}\right] .
\end{aligned}
$$

Notice that Eqs. (18) and (19) applied to homogeneous $\left(\nabla_{a} \varepsilon=\nabla_{a} \mu=0\right)$, isotropic $\left(\chi_{(\varepsilon)}^{a b}=0=\chi_{a b}^{(\mu)}\right)$ materials, with arbitrary four-velocity field $v^{a}$, lead to the same equations which rule minimally coupled vacuum electromagnetism in a curved space-time with metric $\sqrt{\mu / n} g_{a b}$. Optical analogue models in these configurations with $\mu=1$ were studied in $[31,32]$. Here, we shall focus on electromagnetism in anisotropic materials, more specifically, materials with only "shearlike" anisotropies: $\chi_{(\varepsilon)}^{[a b]}=0=\chi_{[a b]}^{(\mu)}$. In this case, the tensor $\chi^{a b c d}$ defined in Eq. (14) has the same algebraic symmetries as the Riemann curvature tensor, namely, $\chi^{\text {abcd }}=$ $\chi^{c d a b}$ and $\chi^{a[b c d]}=0$, in addition to $\chi^{a b c d}=\chi^{[a b][c d]}$, which is always true.

Equations (18) and (19) can be seen as analogous to some nonminimally coupled electromagnetic field equations in curved space-time. Although in general $\chi^{a b c d}$ is independent of the Riemann tensor associated with the effective metric $g_{a b}$, one can construct cases where they are related. This is interesting because some one-loop QED corrections to Maxwell's field equations in curved space-time [33,34] can be emulated by such nonminimal coupling, as we shall discuss below, in Sec. II A.

Before considering particular applications of the equations above, let us define a sesquilinear form on the space of complexified solutions, which will be relevant when applying the canonical quantization procedure. As usual, let us solve Eq. (18) by introducing the four-potential $A_{a}$ such that $F_{a b}=\nabla_{a} A_{b}-\nabla_{b} A_{a}$. Then, let $F_{a b}$ and $F_{a b}^{\prime}$ be two complex solutions of Eq. (19), associated to $A_{a}$ and $A_{a}^{\prime}$, respectively. With overbars representing complex conjugation, we contract
$\bar{A}_{b}$ (respectively, $A_{b}^{\prime}$ ) with Eq. (19) applied to $F_{c d}^{\prime}$ (respectively, $\left.\bar{F}_{c d}\right)$ and subtract one from the other, arriving at

$$
\nabla_{a}\left[\left(g^{a c} g^{b d}+\chi^{a b c d}\right)\left(\bar{A}_{b} F_{c d}^{\prime}-A_{b}^{\prime} \bar{F}_{c d}\right)\right]=0 .
$$

This continuitylike equation ensures that the quantity

$$
\left(A, A^{\prime}\right):=i \int_{\Sigma} d \Sigma N_{a}\left(g^{a c} g^{b d}+\chi^{a b c d}\right)\left(\bar{A}_{b} F_{c d}^{\prime}-A_{b}^{\prime} \bar{F}_{c d}\right)
$$

is independent of the spacelike hypersurface $\Sigma$ where the integration is performed, provided we restrict attention to solutions satisfying "appropriate" boundary condition, where $d \Sigma$ is the physical volume element on $\Sigma$ and $N_{a}=\eta_{a b} N^{b}$, with $N^{a}$ being a unit, future-pointing vector orthogonal to $\Sigma$ (according to $\eta_{a b}$ ). More specifically, considering that the system of interest is contained in the space-time region $\mathcal{M} \cong$ $T \times \Sigma$, where $T \subseteq \mathbb{R}$ is a real open interval, then the appropriate boundary condition amounts to imposing that the flux of the (sesquilinear) current appearing in Eq. (20) vanishes through $T \times \dot{\Sigma}$ (where $\dot{\mathcal{S}}$ denotes the boundary of the space $\mathcal{S})$. In particular, in stationary situations which we shall treat here, this condition translates to

$$
\int_{\dot{\Sigma}} d S s_{a}\left(g^{a c} g^{b d}+\chi^{a b c d}\right)\left(\bar{A}_{b} F_{c d}^{\prime}-A_{b}^{\prime} \bar{F}_{c d}\right)=0,
$$

where $d S$ is the physical area element on $\dot{\Sigma}$ and $s^{a}$ is the unit vector field normal to $T \times \dot{\Sigma}$ (according to $\eta_{a b}$ ). Thus, these conditions being satisfied, Eq. (21) provides a legitimate sesquilinear form on the space $\mathcal{S}_{\mathbb{C}}$ of complex-valued solutions of Eqs. (18) and (19). Notice that for pure-gauge solutions, i.e., $A_{a}=\nabla_{a} \psi$, for some scalar function $\psi-$, $(A, A)=0$. (The converse, however, is not true.)

The relevance of this sesquilinear form is that it provides a legitimate inner product on a (nonunique choice of) subspace $\mathcal{S}_{\mathbb{C}}^{+} \subsetneq \mathcal{S}_{\mathbb{C}}$ of "positive-norm solutions," which, together with its complex conjugate $\mathcal{S}_{\mathbb{C}}^{-} \subsetneq \mathcal{S}_{\mathbb{C}}$, generates all solutions $\mathcal{S}_{\mathbb{C}}: \mathcal{S}_{\mathbb{C}}^{+} \oplus \mathcal{S}_{\mathbb{C}}^{-}=\mathcal{S}_{\mathbb{C}}$. Loosely speaking, upon completion, $\mathcal{S}_{\mathbb{C}}^{+}$ yields a Hilbert space $\mathcal{H}$ from which the (symmetrized) Fock space $\mathcal{F}_{s}(\mathcal{H})$ is canonically constructed to represent states of the electromagnetic field. In particular, choosing $\mathcal{S}_{\mathbb{C}}^{+}$to be generated by positive-frequency solutions (those proportional to $e^{-i \omega t}$, with $\omega>0$ ), the vacuum state of this Fock representation corresponds to the usual physical vacuum state of the field.

\section{A. QED-inspired nonminimal couplings}

As mentioned earlier, Eqs. (18) and (19) can be interpreted as ruling electromagnetism in curved space-times with some QED-inspired nonminimal coupling $\chi^{\text {abcd }}$ with the background geometry. In fact, in the one-loop QED approximation $[33,34]$

$$
\chi^{a b c d}=\alpha_{1} R^{a b c d}+\alpha_{2} R^{[a \mid[c} g^{d] \mid b]}+\alpha_{3} R g^{a[c} g^{d] b},
$$

with $\alpha_{1}=-\alpha_{2} / 13=2 \alpha_{3}=-\alpha /\left(90 \pi m_{e}^{2}\right)$, where $\alpha$ is the fine-structure constant, $m_{e}$ is the electron's mass, and $R^{a b c d}$, $R^{a b}$, and $R$ are, respectively, the Riemann, Ricci, and Ricciscalar curvature tensors associated with the (effective) metric $g_{a b}$. By leaving $\alpha_{1}, \alpha_{2}, \alpha_{3}$ unconstrained, Eq. (23) represents 
a three-parameter family of couplings of the electromagnetic field with the background effective geometry (see Ref. [35] for some interesting particular cases).

For a generic medium, $\chi^{a b c d}$ is not related to the geometry associated with $g_{a b}$. However, we can simulate couplings given by Eq. (23) by conveniently relating $n$ and $v^{a}$ (which determine $g_{a b}$ ) with $\mu$ and the anisotropic tensors $\chi_{(\varepsilon)}^{a b}$ and $\chi_{a b}^{(\mu)}$ (which appear in $\chi^{a b c d}$ ). From Eqs. (14) and (23), and their contractions with $g_{a b}$,

$$
\begin{aligned}
g_{b d} \chi^{a b c d} & =\frac{3}{2}\left(\frac{n}{\mu}-1\right) g^{a c}+\frac{\chi_{(\varepsilon)}^{a c}}{2 n^{3 / 2}}+\frac{n^{3 / 2}}{2} g^{a b} g^{c d} \chi_{b d}^{(\mu)} \\
& =\left(\alpha_{1}+\alpha_{2} / 2\right) R^{a c}+\left(\alpha_{2} / 4+3 \alpha_{3} / 2\right) R g^{a c}, \\
g_{a c} g_{b d} \chi^{a b c d} & =6\left(\frac{n}{\mu}-1\right)=\left(\alpha_{1}+3 \alpha_{2} / 2+6 \alpha_{3}\right) R,
\end{aligned}
$$

we can solve for $\mu$ and the anisotropic tensors, obtaining

$$
\begin{aligned}
\mu= & \frac{n}{1+\left(\alpha_{1} / 6+\alpha_{2} / 4+\alpha_{3}\right) R}, \\
n^{-3 / 2} \chi_{(\varepsilon)}^{a b}= & -2 \alpha_{1}\left(R^{a c b d} V_{c} V_{d}+\frac{R}{12} H^{a b}\right) \\
& +\frac{\alpha_{2}}{2}\left(R^{a b}-\frac{R}{4} g^{a b}\right) \\
n^{3 / 2} \chi_{a b}^{(\mu)}= & 2 \alpha_{1}\left(R_{a c b d} V^{c} V^{d}+\frac{R}{12} H_{a b}\right) \\
& +\frac{\left(4 \alpha_{1}+\alpha_{2}\right)}{2}\left(R^{a b}-\frac{R}{4} g^{a b}\right),
\end{aligned}
$$

where $V^{a}=n^{3 / 4} v^{a}$ is the four-velocity of the medium normalized according to the effective metric $g_{a b}$ and $H_{a b}:=g_{a b}+$ $V_{a} V_{b}$. In Eqs. (27) and (28), indices are lowered and raised by the effective metric and its inverse. Notice that, unless $\alpha_{1}=$ $\alpha_{2}=0$, which implies $\chi_{(\varepsilon)}^{a b}=0=\chi_{a b}^{(\mu)}$, as a consequence of $\chi_{(\varepsilon)}^{a b} v_{b}=0=\chi_{a b}^{(\mu)} v^{b}$, only geometries associated with $g_{a b}$ which can be put in the form given by Eq. (16) and satisfying

$$
R^{a}{ }_{b} v^{b}=\frac{R}{4} v^{a}
$$

for some timelike four-vector $v^{a}$, can be emulated by these anisotropic media, with $v^{a}$ then set as the medium's fourvelocity. Using Einstein's equations to map this constraint to the stress-energy-momentum tensor $T^{a b}$ of the corresponding gravitational source, we have that

$$
T_{b}^{a} v^{b}=\frac{T}{4} v^{a}
$$

where, again, the effective metric and its inverse are used to lower and raise indices (and $T:=T_{a}^{a}$ ). One can easily check that in case of perfect fluids, characterized by a proper energy density $\rho$ and (isotropic) pressure $p$, Eq. (30) is only satisfied for $p=-\rho$, i.e., for a cosmological-constant-type "fluid." However, if one allows for sources with anisotropic pressures $\left(p_{1}, p_{2}, p_{3}\right)$, described by the stress-energy-momentum tensor

$$
T^{a b}=\rho u^{a} u^{b}+\sum_{j=1}^{3} p_{j} \mathbf{e}_{j}^{a} \mathbf{e}_{j}^{b},
$$

with $\left\{u^{a}, \mathbf{e}_{1}^{a}, \mathbf{e}_{2}^{a}, \mathbf{e}_{3}^{a}\right\}$ being a tetrad and $u^{a}$ timelike, then

$$
\rho+\frac{1}{3} \sum_{j=1}^{3} p_{j}=0
$$

and

$$
\left(V_{a} \mathbf{e}_{j}^{a}\right)\left(\rho+p_{j}\right)=0, \quad j=1,2,3 .
$$

In particular, if $V^{a}=u^{a}$, then Eq. (32) is the only additional constraint to be enforced.

Returning attention to the background-effective geometry and recalling that all the geometric tensors are obtained from $g_{a b}$ given in Eq. (16), we see that Eq. (29) actually comprises a system of four differential equations which $n$ and $v^{a}$ must satisfy. Electromagnetism with nonminimal coupling described by Eq. (23) can only be simulated in these anisotropic media if the background space-time geometry is associated to solutions of this system [via Eq. (16)]. We shall treat a particular solution to these differential equations later.

\section{PLANE-SYMMETRIC ANISOTROPIC MEDIUM AT REST}

In this section, we consider the simplest case of an anisotropic medium: a plane-symmetric medium at rest in the inertial laboratory frame. The purpose of this section is not yet to establish an analogy with some interesting gravitational system, but to present the analysis in a simple context. In Sec. IV we apply the analysis to a more appealing scenario.

Let us consider a medium at rest in an inertial laboratory, such that in inertial Cartesian coordinates $\{(t, x, y, z)\}=\mathbb{R}^{3} \times$ $\mathcal{I} \subseteq \mathbb{R}^{4}$ we have $v^{\mu}=(1,0,0,0), \mu=\mu(z), \varepsilon=\varepsilon(z)$,

$$
\chi_{(\varepsilon)}^{\alpha \beta}=\left(\Delta^{(\varepsilon)} / 3\right)\left(2 \delta_{z}^{\alpha} \delta_{z}^{\beta}-\delta_{x}^{\alpha} \delta_{x}^{\beta}-\delta_{y}^{\alpha} \delta_{y}^{\beta}\right),
$$

and

$$
\chi_{\alpha \beta}^{(\mu)}=\left(\Delta^{(\mu)} / 3\right)\left(2 \delta_{\alpha}^{z} \delta_{\beta}^{z}-\delta_{\alpha}^{x} \delta_{\beta}^{x}-\delta_{\alpha}^{y} \delta_{\beta}^{y}\right),
$$

with $\Delta^{(\varepsilon)}=\Delta^{(\varepsilon)}(z), \Delta^{(\mu)}=\Delta^{(\mu)}(z), z \in \mathcal{I}$. $(\mathcal{I}$ is an open real interval.) This simply means that

$$
\begin{aligned}
& D^{j}=\varepsilon_{\perp} E_{j}, \quad j=x, y \\
& D^{z}=\varepsilon_{\|} E_{z}, \\
& H_{j}=\mu_{\perp}^{-1} B^{j}, \quad j=x, y \\
& H_{z}=\mu_{\|}^{-1} B^{z},
\end{aligned}
$$

where $\varepsilon_{\|}-\varepsilon_{\perp} \equiv \Delta^{(\varepsilon)}, \quad\left(2 \varepsilon_{\perp}+\varepsilon_{\|}\right) / 3 \equiv \varepsilon, \quad \mu_{\|}^{-1}-\mu_{\perp}^{-1} \equiv$ $\Delta^{(\mu)}$, and $\left(2 \mu_{\perp}^{-1}+\mu_{\|}^{-1}\right) / 3 \equiv \mu^{-1}$.

In these coordinates, $g_{\mu \nu}=\sqrt{n} \operatorname{diag}\left(-n^{-2}, 1,1,1\right)$. For convenience, we shall work in the generalized Coulomb gauge [36] in which $A_{\mu}=(0, \mathbf{A})$ and $\partial_{\perp} \cdot\left(\varepsilon_{\perp} \mathbf{A}_{\perp}\right)+\partial_{z}\left(\varepsilon_{\|} A_{z}\right)=0$, where we have defined $\mathbf{A}_{\perp}:=\left(A_{x}, A_{y}\right), \partial_{\perp}:=\left(\partial_{x}, \partial_{y}\right)$. In this 
gauge, the $t$ component of Eq. (19) is automatically satisfied, while the spatial components lead to

$$
\begin{aligned}
& {\left[-\frac{\varepsilon_{\perp}}{\mu_{\perp}} \partial_{t}^{2}+\frac{1}{\mu_{\perp} \mu_{\|}} \partial_{\perp}^{2}+\left(\frac{1}{\mu_{\perp}} \partial_{z}\right)^{2}\right] \mathbf{A}_{\perp}} \\
& =\frac{1}{\mu_{\perp} \mu_{\|}}\left[\mu_{\|} \partial_{z}\left(\mu_{\perp}^{-1} \partial_{\perp}\right)-\varepsilon_{\perp}^{-1} \partial_{z}\left(\varepsilon_{\|} \partial_{\perp}\right)\right] A_{z}, \\
& {\left[-\frac{\mu_{\perp}}{\varepsilon_{\perp}} \partial_{t}^{2}+\frac{1}{\varepsilon_{\perp} \varepsilon_{\|}} \partial_{\perp}^{2}+\left(\frac{1}{\varepsilon_{\perp}} \partial_{z}\right)^{2}\right]\left(\varepsilon_{\|} A_{z}\right)=0 .}
\end{aligned}
$$

First, let us consider solutions $\mathbf{A}$ such that $A_{z}=0$, which describe electric fields which are perpendicular to the $z$ direction (transverse electric modes $\mathbf{A}^{(\mathrm{TE})}$, for short [37]). In this case, our gauge condition ensures that there exists a scalar field $\psi$ such that $A_{x}^{(\mathrm{TE})}=\partial_{y} \psi$ and $A_{y}^{(\mathrm{TE})}=-\partial_{x} \psi$. Moreover, making use of the staticity and planar symmetry of the present scenario, we can write $\psi=e^{-i\left(\omega t-\mathbf{k}_{\perp} \cdot \mathbf{x}_{\perp}\right)} f_{\omega k_{\perp}}^{(\mathrm{TE})}(z)$, where $\mathbf{x}_{\perp}=$ $(x, y, 0), \mathbf{k}_{\perp}=\left(k_{x}, k_{y}, 0\right), k_{\perp}=\left\|\mathbf{k}_{\perp}\right\|$, and $f_{\omega k_{\perp}}^{(\mathrm{TE})}(z)$ satisfies

$$
\left[-\frac{d^{2}}{d \zeta^{2}}+\left(\frac{k_{\perp}^{2}}{\mu_{\perp} \mu_{\|}}-\frac{\varepsilon_{\perp} \omega^{2}}{\mu_{\perp}}\right)\right] f_{\omega k_{\perp}}^{(\mathrm{TE})}=0,
$$

with $\zeta$ being a spatial coordinate such that $d \zeta=\mu_{\perp} d z$. Equation (42) must be supplemented by boundary conditions for $f_{\omega k_{\perp}}^{(\mathrm{TE})}$. Imposing Eq. (22) to these modes leads to

$$
\left.\left[\overline{f_{\omega k_{\perp}}^{(\mathrm{TE})}} \frac{d}{d \zeta} f_{\omega^{\prime} k_{\perp}}^{(\mathrm{TE})}-f_{\omega^{\prime} k_{\perp}}^{(\mathrm{TE})} \frac{d}{d \zeta} \overline{f_{\omega k_{\perp}}^{(\mathrm{TE})}}\right]\right|_{\dot{\mathcal{I}}}=0,
$$

where $[\ldots]]_{\dot{\mathcal{I}}}$ denotes the flux of the quantity in square brackets through $\dot{\mathcal{I}}$. This condition restricts the possible values of $\omega^{2}$. Let $\mathcal{E}_{k_{\perp}}^{(\mathrm{TE})}$ be the $\left(k_{\perp}\right.$-dependent) set of $\omega$ values for which Eqs. (42) and (43) are satisfied for $f_{\omega k_{\perp}}^{(\mathrm{TE})} \not \equiv 0$. For $\omega, \omega^{\prime} \in$ $\mathcal{E}_{k_{\perp}+}^{(\mathrm{TE})}:=\mathcal{E}_{k_{\perp}}^{(\mathrm{TE})} \cap \mathbb{R}_{+}^{*}$, we can orthonormalize these modes according to

$$
\begin{aligned}
\left(A_{\omega \mathbf{k}_{\perp}}^{(\mathrm{TE})}, A_{\omega^{\prime} \mathbf{k}_{\perp}^{\prime}}^{(\mathrm{TE})}\right) & =-\left(\overline{A_{\omega \mathbf{k}_{\perp}}^{(\mathrm{TT})}}, \overline{A_{\omega^{\prime} \mathbf{k}_{\perp}^{\prime}}^{(\mathrm{TE})}}\right) \\
& =\delta_{\omega \omega^{\prime}} \delta\left(\mathbf{k}_{\perp}-\mathbf{k}_{\perp}^{\prime}\right), \\
\left(A_{\omega \mathbf{k}_{\perp}}^{(\mathrm{TE})}, \overline{A_{\omega^{\prime} \mathbf{k}_{\perp}^{\prime}}^{(\mathrm{TE})}}\right) & =0
\end{aligned}
$$

$\left(\delta_{\omega \omega^{\prime}}\right.$ being the appropriate Dirac-delta distribution on $\left.\mathcal{E}_{k_{\perp}}^{(\mathrm{TE})}\right)$, where the sesquilinear form given in Eq. (21), applied to the current scenario, takes the form

$$
\begin{aligned}
\left(A, A^{\prime}\right):= & i \int_{\Sigma} d^{3} x\left[\varepsilon_{\perp}\left(\overline{\mathbf{A}}_{\perp} \cdot \partial_{t} \mathbf{A}_{\perp}^{\prime}-\mathbf{A}_{\perp}^{\prime} \cdot \partial_{t} \overline{\mathbf{A}}_{\perp}\right)\right. \\
& \left.+\varepsilon_{\|}\left(\bar{A}_{z} \partial_{t} A_{z}^{\prime}-A_{z}^{\prime} \partial_{t} \bar{A}_{z}\right)\right] .
\end{aligned}
$$

We obtain (up to a global phase)

$$
\mathbf{A}_{\omega \mathbf{k}_{\perp}}^{(\mathrm{TE})}=\frac{\mathbf{k}_{\perp} \times \mathbf{n}_{z}}{2 \pi k_{\perp} \sqrt{2 \omega}} e^{-i\left(\omega t-\mathbf{k}_{\perp} \cdot \mathbf{x}_{\perp}\right)} f_{\omega k_{\perp}}^{(\mathrm{TE})}(z),
$$

with

$$
\int_{\mathcal{I}} d z \varepsilon_{\perp}(z) \overline{f_{\omega k_{\perp}}^{(\mathrm{TE})}}(z) f_{\omega^{\prime} k_{\perp}}^{(\mathrm{TE})}(z)=\delta_{\omega \omega^{\prime}}
$$

and $\mathbf{n}_{z}:=(0,0,1)$.

The second set of solutions of Eqs. (40) and (41), which describe magnetic fields which are perpendicular to the $z$ direction (transverse magnetic modes $\mathbf{A}^{(\mathrm{TM})}$, for short [37]) is obtained by conveniently setting $A_{z}^{(\mathrm{TM})}=\varepsilon_{\|}^{-1} \partial_{\perp}^{2} \phi$, where $\phi$ is an auxiliary function. Our gauge condition then leads to $A_{j}^{(\mathrm{TM})}=-\varepsilon_{\perp}^{-1} \partial_{j} \partial_{z} \phi, j=x, y$. Using, again, staticity and planar symmetry, we find solutions of the form $\phi=$ $e^{-i\left(\omega t-\mathbf{k}_{\perp} \cdot \mathbf{x}_{\perp}\right)} f_{\omega k_{\perp}}^{(\mathrm{TM})}(z)$, where $f_{\omega k_{\perp}}^{(\mathrm{TM})}(z)$ satisfies

$$
\left[-\frac{d^{2}}{d \xi^{2}}+\left(\frac{k_{\perp}^{2}}{\varepsilon_{\perp} \varepsilon_{\|}}-\frac{\mu_{\perp} \omega^{2}}{\varepsilon_{\perp}}\right)\right] f_{\omega k_{\perp}}^{(\mathrm{TM})}=0,
$$

with $\xi$ being a spatial coordinate such that $d \xi=\varepsilon_{\perp} d z$. The boundary condition imposed by Eq. (22) now leads to

$$
\left.\left[\omega^{2} \overline{f_{\omega k_{\perp}}^{(\mathrm{TM})}} \frac{d}{d \xi} f_{\omega^{\prime} k_{\perp}}^{(\mathrm{TM})}-\omega^{\prime 2} f_{\omega^{\prime} k_{\perp}}^{(\mathrm{TM})} \frac{d}{d \xi} \overline{f_{\omega k_{\perp}}^{(\mathrm{TM})}}\right]\right|_{\dot{\mathcal{I}}}=0 .
$$

Let $\mathcal{E}_{k_{\perp}}^{(\mathrm{TM})}$ be the ( $k_{\perp}$-dependent) set of $\omega$ values for which Eqs. (49) and (50) are satisfied for $f_{\omega k_{\perp}}^{(\mathrm{TM})} \not \equiv 0$. For $\omega, \omega^{\prime} \in$ $\mathcal{E}_{k_{\perp}+}^{(\mathrm{TM})}:=\mathcal{E}_{k_{\perp}}^{(\mathrm{TM})} \cap \mathbb{R}_{+}^{*}$, we can normalize these modes according to

$$
\begin{aligned}
\left(A_{\omega \mathbf{k}_{\perp}}^{(\mathrm{TM})}, A_{\left.\omega^{\prime} \mathbf{k}_{\perp}^{\prime}\right)}^{(\mathrm{TM})}\right) & =-\left(\overline{A_{\omega \mathbf{k}_{\perp}}^{(\mathrm{TM})}}, \overline{A_{\omega^{\prime} \mathbf{k}_{\perp}^{\prime}}^{(\mathrm{TM})}}\right) \\
& =\delta_{\omega \omega^{\prime}} \delta\left(\mathbf{k}_{\perp}-\mathbf{k}_{\perp}^{\prime}\right), \\
\left(A_{\omega \mathbf{k}_{\perp}}^{(\mathrm{TM})}, \overline{A_{\left.\omega^{\prime} \mathbf{k}_{\perp}^{\prime}\right)}^{(\mathrm{TM})}}\right) & =0
\end{aligned}
$$

$\left(\delta_{\omega \omega^{\prime}}\right.$ now being the appropriate Dirac-delta distribution on $\mathcal{E}_{k_{\perp}}^{(\mathrm{TM})}$ ), obtaining (up to a global phase)

$$
\mathbf{A}_{\omega \mathbf{k}_{\perp}}^{(\mathrm{TM})}=\frac{e^{-i\left(\omega t-\mathbf{k}_{\perp} \cdot \mathbf{x}_{\perp}\right)}}{2 \pi k_{\perp} \sqrt{2 \omega^{3}}}\left(\frac{k_{\perp}^{2}}{\varepsilon_{\|}} \mathbf{n}_{z}+i \frac{\mathbf{k}_{\perp}}{\varepsilon_{\perp}} \frac{d}{d z}\right) f_{\omega k_{\perp}}^{(\mathrm{TM})}(z),
$$

with

$$
\int_{\mathcal{I}} d z \mu_{\perp}(z) \overline{f_{\omega k_{\perp}}^{(\mathrm{TM})}}(z) f_{\omega^{\prime} k_{\perp}}^{(\mathrm{TM})}(z)=\delta_{\omega \omega^{\prime}}
$$

Moreover, modes $\mathbf{A}_{\omega \mathbf{k}_{\perp}}^{(\mathrm{TM})}$ and $\overline{\mathbf{A}_{\omega \mathbf{k}_{\perp}}^{(\mathrm{TM})}}$ are orthogonal to modes $\mathbf{A}_{\omega \mathbf{k}_{\perp}}^{(\mathrm{TE})}$ and $\overline{\mathbf{A}_{\omega \mathbf{k}_{\perp}}^{(\mathrm{TE})}}$.

The solutions expressed in Eqs. (47) and (53), dubbed positive-frequency normal modes, play a central role in the construction of the Fock (Hilbert) space of the quantized theory, as described at the end of the previous section. With these solutions, the quantum-field operator $\hat{\mathbf{A}}$ is represented by

$$
\hat{\mathbf{A}}=\sum_{\mathrm{J} \in\{\mathrm{TE}, \mathrm{TM}\}} \int_{\mathbb{R}^{2}} d^{2} \mathbf{k}_{\perp} \int_{\mathcal{E}_{k_{\perp}}^{(\mathrm{J})}} d \omega\left[\hat{a}_{\omega \mathbf{k}_{\perp}}^{(\mathrm{J})} \mathbf{A}_{\omega \mathbf{k}_{\perp}}^{(\mathrm{J})}+\text { H.c. }\right],
$$

where "H.c." stands for "Hermitian conjugate" of the preceding term and $\hat{a}_{\omega \mathbf{k}_{\perp}}^{(\mathrm{J})}$ (respectively, $\hat{a}_{\omega \mathbf{k}_{\perp}}^{(\mathrm{J} \dagger}$ ) is the annihilation (respectively, creation) operator associated with mode $\mathbf{A}_{\omega \mathbf{k}_{\perp}}^{(\mathrm{J})}$, satisfying the canonical commutation relations:

$$
\begin{aligned}
& {\left[\hat{a}_{\omega \mathbf{k}_{\perp}}^{(\mathrm{J})}, \hat{a}_{\omega^{\prime} \mathbf{k}_{\perp}^{\prime}}^{\left(\mathrm{J}^{\prime} \dagger\right.}\right]=\delta^{\mathrm{J} J^{\prime}} \delta_{\omega \omega^{\prime}} \delta\left(\mathbf{k}_{\perp}-\mathbf{k}_{\perp}^{\prime}\right),} \\
& {\left[\hat{a}_{\omega \mathbf{k}_{\perp}}^{(\mathrm{J})}, \hat{a}_{\omega^{\prime} \mathbf{k}_{\perp}^{\prime}}^{\left(\mathrm{J}^{\prime}\right)}=0 .\right.}
\end{aligned}
$$

As an application of our quantization scheme, one can use the above formulas to obtain, for instance, the CarnigliaMandel quantization [38] in a straightforward way. The system in this case is composed by a dielectric-vacuum interface 
at $z=0$ and a nonmagnetizable $\left(\mu_{\|}=\mu_{\perp}=1\right)$ homogeneous isotropic nondispersive dielectric $\left(\varepsilon_{\|}=\varepsilon_{\perp}=\varepsilon \equiv n^{2}\right)$ filling the half-space $z<0$. These data enter Eqs. (42) and (49), thus describing the background in terms of effective potentials of one-dimensional Schrödinger-type problems.

\section{A. Instability analysis}

In the analysis presented above, it was implicitly assumed that all constitutive functions $\varepsilon_{\perp}, \varepsilon_{\|}, \mu_{\perp}$, and $\mu_{\|}$are positive functions of $z \in \mathcal{I}$. This condition ensures that the field modes presented in Eqs. (47) and (53), together with their complex conjugates, constitute a complete set of (complexified) solutions of Maxwell equations in $\mathbb{R}^{3} \times \mathcal{I}$; in other words, the boundary-value problems defined by Eqs. (42) and (43) and (49) and (50) admit solutions only for (a subset of) $\omega^{2}>0$. This is easily seen by interpreting them as null-eigenvalue problems for the linear operators defined in the square brackets of Eqs. (42) and (49). Experience with Schrödinger-type equations teaches us that these equations have solutions provided the associated effective potentials (terms in parentheses) become sufficiently negative in a given region, which implies $\omega^{2}>0$ and, typically, the larger the $k_{\perp}^{2}$, the larger the $\omega^{2}$.

Here, however, we shall consider a more interesting situation. It has been known for almost two decades that materials can be engineered so that some of their constitutive functions can assume negative values [39-43]. These exotic materials have been termed metamaterials. In this case, the effective potentials appearing in Eqs. (42) and (49) may become sufficiently negative, granting solutions to these boundary-value problems, without demanding $\omega^{2}>0$. For instance, if $\mu_{\|}<0$ (with $\mu_{\perp}, \varepsilon_{\perp}>0$ ), then the larger the value of $k_{\perp}$, the more negatively it contributes to the effective potential of Eq. (42), favoring the appearance of solutions with smaller (possibly negative) values of $\omega^{2}$. The same is true for Eq. (49) if $\varepsilon_{\|}<0$ and similar analysis can be done if any other constitutive function becomes negative.

At this point, we must introduce an element of reality concerning the constitutive functions. We have been treating these quantities as given functions of $z$ alone, neglecting dispersion effects, since we are, here, interested in gravity analogues. However, these material properties generally depend on characteristics of the electromagnetic field itself, particularly on its time variation (i.e., on $\omega$ ), in which case Eqs. (7) and (8) would be valid mode by mode, with the constitutive tensors $\varepsilon^{a b}$ and $\mu_{a b}$ possibly being different for different modes. When translated to space-time-dependent quantities, Eqs. (7) and (8) would be substituted by sums over the set of allowed field modes [44].

Therefore, the precise key assumption about our metamaterial media is that some of their anisotropic constitutive functions $\varepsilon_{\perp}, \varepsilon_{\|}, \mu_{\perp}, \mu_{\|}$can become negative for some $\omega$ on the positive imaginary axis $\omega^{2}<0$. Notwithstanding, the less restrictive condition $\operatorname{Im}(\omega)>0$ would suffice for our purposes. However, dealing with the case $\operatorname{Im}(\omega) \operatorname{Re}(\omega) \neq 0$ would involve quantization in active media, which we shall treat elsewhere [44]. Moreover, our focus here is to show that the electromagnetic field itself can exhibit interesting behavior without need to exchange energy with the medium (which occurs in dispersive/active media). This justifies our focus on $\omega^{2}<0$ in what follows. The possibility of having this type of material will be discussed later.

Let $\omega^{2}=-\Omega^{2}$ (with $\Omega>0$ ) be such value for which at least one of the constitutive functions is negative for $z \in \mathcal{I}$. Thus, both the effective potentials of Eqs. (42) and (49) take the general form

$$
V_{\mathrm{eff}}=C_{1} k_{\perp}^{2}+C_{2} \Omega^{2}
$$

with $C_{1}$ and $C_{2}$ being functions of $z$. Two interesting possibilities arise:

(i) $C_{1}<0$ : In this case, the larger the value of $k_{\perp}$, the more negative the effective potential gets. Therefore, it is quite reasonable to expect that, for a given size of the interval $\mathcal{I}$, one can always find "large enough" values of $k_{\perp}$ (certainly satisfying $k_{\perp}^{2}>C_{2} \Omega^{2} /\left|C_{1}\right|$ ) such that the Schrödinger-type equation with effective potential $V_{\text {eff }}$ admits null-eigenvalue solutions. We shall refer to this situation as large- $k_{\perp}$ instability.

(ii) $C_{1}>0$ and $C_{2}<0$ : Under these conditions, the effective potential $V_{\text {eff }}$, as a function of $k_{\perp}$, is bounded from below: $V_{\text {eff }} \geqslant-\left|C_{2}\right| \Omega^{2}$. Therefore, a Schrödinger-type equation with effective potential $V_{\text {eff }}$ only admits null-eigenvalue solutions provided $k_{\perp}$ is 'sufficiently small' (certainly satisfying $k_{\perp}^{2}<$ $\left.\left|C_{2}\right| \Omega^{2} / C_{1}\right)$ and the size of the interval where $V_{\text {eff }}$ is negative is "sufficiently large." We shall refer to this situation as minimum-width instability.

Let us call $g_{\Omega k_{\perp}}^{(\mathrm{J})}$ the null-eigenvalue solutions mentioned in either case above, with $\mathrm{J} \in\{\mathrm{TE}, \mathrm{TM}\}$ depending on whether it refers to Eq. (42) or (49) with $\omega^{2}=-\Omega^{2}$ (without loss of generality, $\Omega>0$ ). These solutions are associated with unstable electromagnetic modes whose temporal behavior is proportional to $e^{ \pm \Omega t}$. Although it might be tempting not to consider these "runaway" solutions [25,26], they are essential, if they exist, to expand an arbitrary initial field configuration satisfying the boundary-value problems set by Eqs. (42) and (43) and (49) and (50); in other words, the stationary modes alone do not constitute a complete set of solutions of Maxwell's equations with the given boundary conditions. And even if, on the classical level, one might want to restrict attention to initial field configurations which have no contribution coming from these unstable modes, which is certainly unnatural, for causality forbids the system to constrain its initial configuration based on its future behavior, inevitable quantum fluctuations of these modes would grow, making them dominant some time $e$-foldings $\left(t \sim N \Omega^{-1}, N \gg 1\right)$ after the proper material conditions having been engineered. Therefore, these modes are as physical as the oscillatory ones. In fact, artificial inconsistencies have been reported in the literature, regarding field quantization in active media $[25,26]$, which are completely cured when unstable modes are included in the analysis [44].

It is interesting to note that depending on which constitutive function is negative, Eqs. (42) and (49) may incur different types of instabilities. For instance, if $\mu_{\perp}<0$ for a given $\omega^{2}=-\Omega^{2}<0$, with all other constitutive functions being positive, then Eq. (42) exhibits case (i) instability, while Eq. (49) incurs in case (ii) instability. This means that unstable TE modes, with some $k_{\perp}>\sqrt{\mu_{\|} \varepsilon_{\perp}} \Omega$, would certainly be present, while unstable TM modes, with some $k_{\perp}<\sqrt{\left|\mu_{\perp}\right| \varepsilon_{\|}} \Omega$, would only appear if the width of the 
material (size of the interval $\mathcal{I}$ ) is larger than some critical value. We shall illustrate these facts in a simple example below. But first, let us analyze some features of these unstable modes. In order not to rely on particular initial field configurations, let us focus on the inevitable quantum fluctuations of these modes.

\section{Unstable TE modes}

Repeating the procedure which led us from Eq. (42) to Eq. (47) for the stable modes, unstable TE modes $A_{\Omega \mathbf{k}_{\perp}}^{(u \mathrm{TE})}$ properly orthonormalized according to

$$
\begin{aligned}
\left(A_{\Omega \mathbf{k}_{\perp}}^{(u \mathrm{TE})}, A_{\Omega^{\prime} \mathbf{k}_{\perp}^{\prime}}^{(u \mathrm{TE})}\right) & =-\left(\overline{A_{\Omega \mathbf{k}_{\perp}}^{(u \mathrm{TE})}}, \overline{A_{\left.\Omega^{\prime} \mathbf{k}_{\perp}^{\prime}\right)}^{(u \mathrm{TE})}}\right) \\
& =\delta_{\Omega \Omega^{\prime}} \delta\left(\mathbf{k}_{\perp}-\mathbf{k}_{\perp}^{\prime}\right), \\
\left(A_{\Omega \mathbf{k}_{\perp}}^{(u \mathrm{TE})}, \overline{A_{\left.\Omega^{\prime} \mathbf{k}_{\perp}^{\prime}\right)}^{(u \mathrm{TE})}}\right) & =0
\end{aligned}
$$

(and orthogonal to all other modes) read as (up to a time translation)

$$
\begin{aligned}
\mathbf{A}_{\Omega \mathbf{k}_{\perp}}^{(u \mathrm{TE})}= & \frac{\mathbf{k}_{\perp} \times \mathbf{n}_{z}}{4 \pi k_{\perp} \sqrt{\Omega \sin \kappa}} e^{i \mathbf{k}_{\perp} \cdot \mathbf{x}_{\perp}} g_{\Omega k_{\perp}}^{(\mathrm{TE})}(z) \\
& \times\left(e^{\Omega t-i s_{\varepsilon}^{\perp} \kappa / 2}+e^{-\Omega t+i s_{\varepsilon}^{\perp} \kappa / 2}\right),
\end{aligned}
$$

with $0<\kappa<\pi, g_{\Omega k_{\perp}}^{(\mathrm{TE})}$ normalized according to

$$
\left|\int_{\mathcal{I}} d z \varepsilon_{\perp}(z) \overline{g_{\Omega k_{\perp}}^{(\mathrm{TE})}}(z) g_{\Omega^{\prime} k_{\perp}}^{(\mathrm{TE})}(z)\right|=\delta_{\Omega \Omega^{\prime}}
$$

and $s_{\varepsilon}^{\perp}$ being the sign of the integral above. Calculating the electric $\mathbf{E}_{\Omega \mathbf{k}_{\perp}}^{(u \mathrm{TE})}$ and magnetic $\mathbf{B}_{\Omega \mathbf{k}_{\perp}}^{(u \mathrm{TE})}$ fields associated to these modes, we have

$$
\begin{aligned}
\mathbf{E}_{\Omega \mathbf{k}_{\perp}}^{(u \mathrm{TE})}= & \frac{\sqrt{\Omega}}{4 \pi k_{\perp} \sqrt{\sin \kappa}}\left(\mathbf{n}_{z} \times \mathbf{k}_{\perp}\right) e^{i \mathbf{k}_{\perp} \cdot \mathbf{x}_{\perp}} g_{\Omega k_{\perp}}^{(\mathrm{TE})}(z) \\
& \times\left(e^{\Omega t-i s_{\varepsilon}^{\perp} \kappa / 2}-e^{-\Omega t+i s_{\varepsilon}^{\perp} \kappa / 2}\right), \\
\mathbf{B}_{\Omega \mathbf{k}_{\perp}}^{(u \mathrm{TE})}= & \frac{e^{i \mathbf{k}_{\perp} \cdot \mathbf{x}_{\perp}}}{4 \pi k_{\perp} \sqrt{\Omega \sin \kappa}}\left(-i k_{\perp}^{2} \mathbf{n}_{z}+\mathbf{k}_{\perp} \frac{d}{d z}\right) g_{\Omega k_{\perp}}^{(\mathrm{TE})}(z) \\
& \times\left(e^{\Omega t-i s_{\varepsilon}^{\perp} \kappa / 2}+e^{-\Omega t+i s_{\varepsilon}^{\perp} \kappa / 2}\right) .
\end{aligned}
$$

\section{Unstable TM modes}

Now, turning to the TM modes, we repeat the procedure which led us from Eq. (49) to Eq. (53) for the stable modes. Unstable TM modes $A_{\Omega \mathbf{k}_{\perp}}^{(u \mathrm{TM})}$ properly orthonormalized according to

$$
\begin{aligned}
\left(A_{\Omega \mathbf{k}_{\perp}}^{(u \mathrm{TM})}, A_{\Omega^{\prime} \mathbf{k}_{\perp}^{\prime}}^{(u \mathrm{TM})}\right) & =-\left(\overline{A_{\Omega \mathbf{k}_{\perp}}^{(u \mathrm{TM})}}, \overline{A_{\left.\Omega^{\prime} \mathbf{k}_{\perp}^{\prime}\right)}^{(u \mathrm{TM})}}\right) \\
& =\delta_{\Omega \Omega^{\prime}} \delta\left(\mathbf{k}_{\perp}-\mathbf{k}_{\perp}^{\prime}\right), \\
\left(A_{\Omega \mathbf{k}_{\perp}}^{(u \mathrm{TM})}, \overline{A_{\Omega^{\prime} \mathbf{k}_{\perp}^{\prime}}^{(u \mathrm{TM})}}\right) & =0
\end{aligned}
$$

(and orthogonal to all other modes) read as (up to a time translation)

$$
\begin{aligned}
\mathbf{A}_{\Omega \mathbf{k}_{\perp}}^{(u \mathrm{TM})}= & \frac{e^{i \mathbf{k}_{\perp} \cdot \mathbf{x}_{\perp}}}{4 \pi k_{\perp} \sqrt{\Omega^{3} \sin \kappa}}\left(\frac{k_{\perp}^{2}}{\varepsilon_{\|}} \mathbf{n}_{z}+i \frac{\mathbf{k}_{\perp}}{\varepsilon_{\perp}} \frac{d}{d z}\right) g_{\Omega k_{\perp}}^{(\mathrm{TM})}(z) \\
& \times\left(e^{\Omega t+i s_{\mu}^{\perp} \kappa / 2}+e^{-\Omega t-i s_{\mu}^{\perp} \kappa / 2}\right),
\end{aligned}
$$

where, again, $0<\kappa<\pi, g_{\Omega k_{\perp}}^{(\mathrm{TM})}$ is normalized according to

$$
\left|\int_{\mathcal{I}} d z \mu_{\perp}(z) \overline{g_{\Omega k_{\perp}}^{(\mathrm{TM})}}(z) g_{\Omega^{\prime} k_{\perp}}^{(\mathrm{TM})}(z)\right|=\delta_{\Omega \Omega^{\prime}},
$$

and $s_{\mu}^{\perp}$ is the sign of the integral above. Calculating the electric $\mathbf{E}_{\Omega \mathbf{k}_{\perp}}^{(u \mathrm{TM})}$ and magnetic $\mathbf{B}_{\Omega \mathbf{k}_{\perp}}^{(u \mathrm{TM})}$ fields associated to these modes, we have

$$
\begin{aligned}
\mathbf{E}_{\Omega \mathbf{k}_{\perp}}^{(u \mathrm{TM})}= & -\frac{e^{i \mathbf{k}_{\perp} \cdot \mathbf{x}_{\perp}}}{4 \pi k_{\perp} \sqrt{\Omega \sin \kappa}}\left(\frac{k_{\perp}^{2}}{\varepsilon_{\|}} \mathbf{n}_{z}+i \frac{\mathbf{k}_{\perp}}{\varepsilon_{\perp}} \frac{d}{d z}\right) g_{\Omega k_{\perp}}^{(\mathrm{TM})}(z) \\
& \times\left(e^{\Omega t+i s_{\mu}^{\perp} \kappa / 2}-e^{-\Omega t-i s_{\mu}^{\perp} \kappa / 2}\right), \\
\mathbf{B}_{\Omega \mathbf{k}_{\perp}}^{(u \mathrm{TM})}= & \frac{i \mu_{\perp} \sqrt{\Omega}}{4 \pi k_{\perp} \sqrt{\sin \kappa}}\left(\mathbf{n}_{z} \times \mathbf{k}_{\perp}\right) e^{i \mathbf{k}_{\perp} \cdot \mathbf{x}_{\perp}} g_{\Omega k_{\perp}}^{(\mathrm{TM})}(z) \\
& \times\left(e^{\Omega t+i s_{\mu}^{\perp} \kappa / 2}+e^{-\Omega t-i s_{\mu}^{\perp} \kappa / 2}\right) .
\end{aligned}
$$

The modes given by Eqs. (61) and (67), if present, must be added to the expansion of the field operator $\hat{\mathbf{A}}$ given in Eq. (55), along with their complex conjugates, with corresponding annihilation $\hat{a}_{\Omega \mathbf{k}_{\perp}}^{(u \mathrm{~J})}$ and creation $\hat{a}_{\Omega \mathbf{k}_{\perp}}^{(u \mathrm{~J})}$ operators, $\mathrm{J} \in\{\mathrm{TE}, \mathrm{TM}\}$. The resulting operator expansion can then be used to calculate electromagnetic-field fluctuations and correlations. In the presence of unstable modes, it is easy to see that the field's vacuum fluctuations are eventually $\left(t \gg \Omega^{-1}\right)$ dominated by these exponentially growing modes. Obviously, this instability cannot persist indefinitely as these wild fluctuations will affect the medium's properties, supposedly leading the whole system to a final stable state. In some gravitational contexts, stabilization occurs by decoherence of these growing vacuum fluctuations [13], giving rise to a nonzero classical field configuration, a phenomenon called spontaneous scalarization (for spin-0) [14-17] or vectorization (for spin-1 fields) [18]. It is possible that something similar might occur in the analogous system.

We shall discuss this point further in Sec. V.

\section{B. Example}

Let us consider a very simple system just to illustrate the results above in a concrete scenario: a slab of width $L$ (in the region $-L / 2<z<L / 2$ ), made of a homogeneous material with, say, $\mu_{\perp}<0$ for a given $\omega^{2}=-\Omega^{2}(\Omega>0)$ and all other constitutive functions positive. For concreteness sake, here we assume that this value $\omega^{2}=-\Omega^{2}$ is isolated and that it is the most negative value of $\omega^{2}$ for which $\mu_{\perp}<0$. This latter assumption is merely a matter of choice, while the former only affects the measure on the set of quantum numbers $\mathbf{k}_{\perp}: \int d^{2} \mathbf{k}_{\perp} \rightarrow \int d \theta \sum_{k_{\perp}} 2 \pi k_{\perp} / L_{\perp}, \delta\left(\mathbf{k}_{\perp}-\mathbf{k}_{\perp}^{\prime}\right) \rightarrow$ $L_{\perp} \delta_{k_{\perp} k_{\perp}^{\prime}} \delta\left(\theta-\theta^{\prime}\right) /\left(2 \pi k_{\perp}\right)$, where $L_{\perp}$ is the length scale associated with the area of the "infinite" slab $\left(L_{\perp} \gg L\right)$.

According to the discussion presented earlier, in this scenario, TE modes incur in case (i) (large- $\left.k_{\perp}\right)$ instability, while TM modes undergo case (ii) (minimum-width) instability. The 


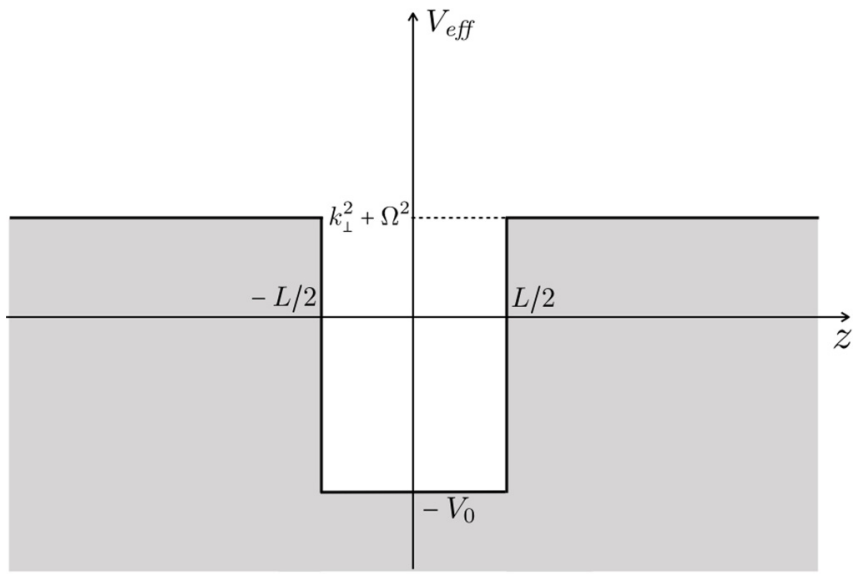

FIG. 1. Effective potential well which represents the homogeneous slab with negative $\mu_{\perp}$ for the unstable electromagnetic modes.

solutions $g_{\Omega k_{\perp}}^{(\mathrm{J})}$ of Eqs. (42) and (49) with $\omega^{2}=-\Omega^{2}$ are given by the normalizable [according to Eqs. (62) and (68)] solutions of the null-eigenvalue, Schrödinger-type equation

$$
\left(-\frac{d^{2}}{d z^{2}}+V_{\text {eff }}\right) g_{\Omega k_{\perp}}^{(\mathrm{J})}=0,
$$

with $V_{\text {eff }}$ being the well potential represented in Fig. 1. The depth of the potential is given by

$$
V_{0}= \begin{cases}\left|\mu_{\perp}\right| \varepsilon_{\perp} \Omega^{2}+\frac{\left|\mu_{\perp}\right|}{\mu_{\|}} k_{\perp}^{2}, & \mathrm{~J}=\mathrm{TE} \\ \left|\mu_{\perp}\right| \varepsilon_{\perp} \Omega^{2}-\frac{\varepsilon_{\perp}}{\varepsilon_{\|}} k_{\perp}^{2}, & \mathrm{~J}=\mathrm{TM} .\end{cases}
$$

Although here we focus only on unstable modes, associated with $g_{\Omega k_{\perp}}^{(J)}$, note that in this example there would also appear stationary bound solutions associated with $f_{\omega_{0} k_{\perp}}^{(\mathrm{TE})}$ (if $\mu_{\perp}<0$ for some $\omega_{0} \in \mathbb{R}$ ), for some $k_{\perp}^{2}>\max \left\{\omega_{0}^{2},\left(n_{\perp}^{(\mathrm{TE})} \omega_{0}\right)^{2}\right\}$, where $n_{\perp}^{(\mathrm{TE})}:=\sqrt{\mu_{\|} \varepsilon_{\perp}}$ is the transverse refractive index for the TE modes. For such a hypothetical mode, the slab would act as a waveguide, keeping the mode confined due to total internal reflections at its boundaries. The only peculiar feature here is that $k_{\perp}$ would assume arbitrarily large values (in practice, limited only by the inverse length scale below which the continuous-medium idealization breaks down) for a given $\omega_{0}$.

Back to the unstable modes, a straightforward calculation leads to the familiar even and odd solutions to the square-well potential, with $g_{\Omega k_{\perp}}^{(\mathrm{J})}(z)$ exponentially suppressed for $|z|>L / 2$ and

$$
g_{\Omega k_{\perp}}^{(\mathrm{J})}(z)=\left\{\begin{array}{l}
\frac{\mathcal{N}_{m}^{(J)}}{\cos a_{m}} \cos \left(2 a_{m} z / L\right), \quad 0 \leqslant m \text { even } \\
\frac{\mathcal{N}_{m}^{(J)}}{\sin a_{m}} \sin \left(2 a_{m} z / L\right), \quad 1 \leqslant m \text { odd }
\end{array}\right.
$$

$(-L / 2 \leqslant z \leqslant L / 2)$, where $\mathcal{N}_{m}^{(\mathrm{J})}$ are normalization constants and, for the TE modes, $a_{m} \geqslant \Omega L\left|n_{\|}\right| / 2$ (with $n_{\|}^{2}:=\mu_{\perp} \varepsilon_{\perp}$ ) are solutions of the transcendental equations

$$
\begin{aligned}
& \sqrt{\left|\mu_{\perp}\right| \mu_{\|}} \sqrt{1-\frac{\Omega^{2} L^{2}}{4 a_{m}^{2}}\left|n_{\|}^{2}\right|\left[1-\left(n_{\perp}^{(\mathrm{TE})}\right)^{-2}\right]} \\
& = \begin{cases}-\tan a_{m}, & m \text { even } \\
\cot a_{m}, & m \text { odd }\end{cases}
\end{aligned}
$$

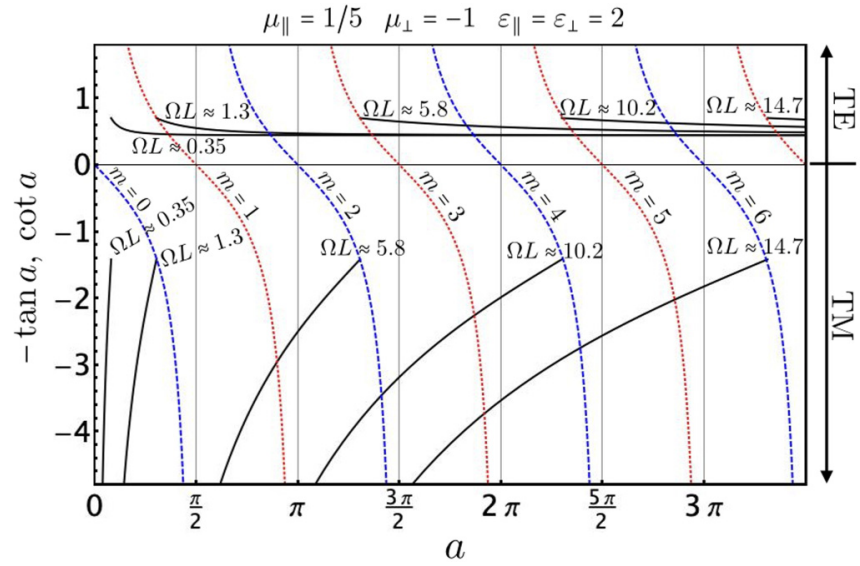

FIG. 2. Graphic representation of solutions of Eqs. (73) and (74). The solid black curves in the upper (respectively, lower) half plane represent the left-hand side (lhs) of Eq. (73) [respectively, minus the lhs of Eq. (74)], with $a_{m}$ replaced by the variable $a$, for different values of $\Omega L$. The dashed blue lines (respectively, dotted red lines) represent the function $-\tan a$ (respectively, $\cot a$ ). The values $a_{m}$ appearing in Eq. (72) are determined by the crossing of the corresponding solid black curve with the dashed blue lines (for $m$ even) and the dotted red lines (for $m$ odd).

while for the TM modes, $0 \leqslant a_{m} \leqslant \Omega L\left|n_{\|}\right| / 2$ and

$$
\begin{aligned}
& \sqrt{\varepsilon_{\perp} \varepsilon_{\|}} \sqrt{\frac{\Omega^{2} L^{2}}{4 a_{m}^{2}}\left|n_{\|}^{2}\right|\left[1+\left|n_{\perp}^{(\mathrm{TM})}\right|^{-2}\right]-1} \\
& = \begin{cases}\tan a_{m}, & m \text { even } \\
-\cot a_{m}, & m \text { odd. }\end{cases}
\end{aligned}
$$

The transverse momentum $k_{\perp}$ is given in terms of $a_{m}$ by

$$
k_{\perp}=k_{\perp}^{(m)}:= \begin{cases}\frac{2}{L} \sqrt{\frac{\mu_{\|}}{\left|\mu_{\perp}\right|}\left(a_{m}^{2}-\left|n_{\|}^{2}\right| \frac{\Omega^{2} L^{2}}{4}\right)}, & \text { TE modes } \\ \frac{2}{L} \sqrt{\frac{\varepsilon_{\|}}{\varepsilon_{\perp}}\left(\left|n_{\|}^{2}\right| \frac{\Omega^{2} L^{2}}{4}-a_{m}^{2}\right)}, & \text { TM modes. }\end{cases}
$$

The explicit form of $\mathcal{N}_{m}^{(\mathrm{J})}$ is not particularly important, so we only present its asymptotic behavior for $k_{\perp} \rightarrow \infty$ for the TE modes

$$
\mathcal{N}_{m}^{(\mathrm{TE})} \approx\left\{\begin{array}{ll}
\sqrt{\frac{2\left(1+\left|\mu_{\perp}\right| \mu_{\|}\right)}{L \varepsilon_{\perp}}}, & m \text { even } \\
\sqrt{\frac{2\left(1+\left|\mu_{\perp}\right| \mu_{\|}\right)}{L \varepsilon_{\perp}\left|\mu_{\perp}\right| \mu_{\|}}}, & m \text { odd }
\end{array}, k_{\perp} \gg \Omega\right.
$$

and for $k_{\perp} \rightarrow 0$ for both TE and TM modes

$$
\begin{aligned}
& \mathcal{N}_{m}^{(\mathrm{TE})} \approx\left\{\begin{array}{ll}
\sqrt{\frac{2\left(\varepsilon_{\perp}+\left|\mu_{\perp}\right|\right)}{L \varepsilon_{\perp}^{2}}}, & m \text { even } \\
\sqrt{\frac{2\left(\varepsilon_{\perp}+\left|\mu_{\perp}\right|\right)}{L \varepsilon_{\perp}\left|\mu_{\perp}\right|}}, & m \text { odd }
\end{array}, k_{\perp} \ll \Omega\right. \\
& \mathcal{N}_{m}^{(\mathrm{TM})} \approx\left\{\begin{array}{ll}
\sqrt{\frac{2\left(\varepsilon_{\perp}+\left|\mu_{\perp}\right|\right)}{L\left|\mu_{\perp}\right|^{2}}}, & m \text { even } \\
\sqrt{\frac{2\left(\varepsilon_{\perp}+\left|\mu_{\perp}\right|\right)}{L \varepsilon_{\perp}\left|\mu_{\perp}\right|}}, & m \text { odd }
\end{array}, k_{\perp} \ll \Omega .\right.
\end{aligned}
$$

In Fig. 2, we plot, for different values of $\Omega L$ and given values of $\mu_{\|}, \mu_{\perp}, \varepsilon_{\|}$, and $\varepsilon_{\perp}$, the left-hand side of Eq. (73) (solid black curves in the upper half plane) minus the left-hand side of Eq. (74) (solid black curves in the lower half plane), substituting, in both $a_{m}$ by the variable $a$ and the functions $-\tan a$ and $\cot a$ (blue dashed lines and red dotted lines, 
respectively). Crossing of the blue dashed lines (respectively, red dotted lines) with a fixed solid black curve determines values $a=a_{m}$ for even (respectively, odd) solutions $g_{\Omega k}^{(\mathrm{J})}$, for the corresponding value of $\Omega L$. The figure clearly corroborates our preliminary analysis, showing that unstable TE modes appear with arbitrarily large values of $a_{m}$ (and, therefore, of $k_{\perp}$ ) and that unstable TM modes only appear if $L$ is larger than some minimum width $L_{0}$, given by

$$
L_{0}=\frac{2 \Omega^{-1}}{\left|n_{\|}\right|} \tan ^{-1}\left(\sqrt{\frac{\varepsilon_{\perp}}{\left|\mu_{\perp}\right|}}\right) .
$$

The unstable TE and TM modes inside the slab can then be put in the form

$$
\begin{aligned}
\mathbf{A}_{\Omega \mathbf{k}_{\perp}^{(m)}}^{(u \mathrm{TE})}= & \frac{\mathcal{N}_{m}^{(\mathrm{TE})}\left(\mathbf{k}_{\perp}^{(m)} \times \mathbf{n}_{z}\right)}{4 \pi k_{\perp}^{(m)} \sqrt{\Omega \sin \kappa_{m}}} \frac{\cos \left(2 a_{m} z / L+m \pi / 2\right)}{\cos \left(a_{m}+m \pi / 2\right)} e^{i \mathbf{k}_{\perp}^{(m)} \cdot \mathbf{x}_{\perp}}\left(e^{\Omega t-i \kappa_{m} / 2}+e^{-\Omega t+i \kappa_{m} / 2}\right), \quad m \geqslant m^{(\mathrm{TE})} \\
\mathbf{A}_{\Omega \mathbf{k}_{\perp}^{(m)}}^{(u \mathrm{TM})}= & \frac{\mathcal{N}_{m}^{(\mathrm{TM})} e^{i \mathbf{k}_{\perp}^{(m)} \cdot \mathbf{x}_{\perp}}}{4 \pi \sqrt{\Omega^{3} \sin \kappa_{m}}}\left(e^{\Omega t-i \kappa_{m} / 2}+e^{-\Omega t+i \kappa_{m} / 2}\right) \\
& \times\left[\frac{k_{\perp}^{(m)} \mathbf{n}_{z}}{\varepsilon_{\|}} \frac{\cos \left(2 a_{m} z / L+m \pi / 2\right)}{\cos \left(a_{m}+m \pi / 2\right)}-i \frac{\mathbf{k}_{\perp}^{(m)}}{\varepsilon_{\perp} k_{\perp}^{(m)}} \frac{2 a_{m}}{L} \frac{\sin \left(2 a_{m} z / L+m \pi / 2\right)}{\cos \left(a_{m}+m \pi / 2\right)}\right], \quad 0 \leqslant m \leqslant m^{(\mathrm{TM})}
\end{aligned}
$$

with

$$
\begin{aligned}
m^{(\mathrm{TE})} & :=\left[1+\left(\frac{L}{L_{0}}-1\right) \frac{2}{\pi} \tan ^{-1}\left(\sqrt{\frac{\varepsilon_{\perp}}{\left|\mu_{\perp}\right|}}\right)\right\rceil, \\
m^{(\mathrm{TM})} & :=\left\lfloor\left(\frac{L}{L_{0}}-1\right) \frac{2}{\pi} \tan ^{-1}\left(\sqrt{\frac{\varepsilon_{\perp}}{\left|\mu_{\perp}\right|}}\right)\right\rfloor
\end{aligned}
$$

( $\lceil x\rceil$ represents the smallest integer larger than, or equal to, $x$, while $\lfloor x\rfloor$ represents the largest integer smaller than, or equal to, $x$ ). The corresponding electric and magnetic field modes are

$$
\begin{aligned}
& \mathbf{E}_{\Omega \mathbf{k}_{\perp}^{(m)}}^{(u \mathrm{TE})}=\frac{\mathcal{N}_{m}^{(\mathrm{TE})}\left(\mathbf{n}_{z} \times \mathbf{k}_{\perp}^{(m)}\right) \sqrt{\Omega}}{4 \pi k_{\perp}^{(m)} \sqrt{\sin \kappa_{m}}} \frac{\cos \left(2 a_{m} z / L+m \pi / 2\right)}{\cos \left(a_{m}+m \pi / 2\right)} e^{i \mathbf{k}_{\perp}^{(m)} \cdot \mathbf{x}_{\perp}}\left(e^{\Omega t-i \kappa_{m} / 2}-e^{-\Omega t+i \kappa_{m} / 2}\right), \quad m \geqslant m^{(\mathrm{TE})} \\
& \mathbf{B}_{\Omega \mathbf{k}_{\perp}^{(m)}}^{(u \mathrm{TE})}=\frac{\mathcal{N}_{m}^{(\mathrm{TE})} e^{i \mathbf{k}_{\perp} \cdot \mathbf{x}_{\perp}}}{4 \pi \sqrt{\Omega \sin \kappa_{m}}}\left(e^{\Omega t-i \kappa_{m} / 2}+e^{-\Omega t+i \kappa_{m} / 2}\right) \\
& \times\left[-i k_{\perp}^{(m)} \mathbf{n}_{z} \frac{\cos \left(2 a_{m} z / L+m \pi / 2\right)}{\cos \left(a_{m}+m \pi / 2\right)}-\frac{\mathbf{k}_{\perp}^{(m)}}{k_{\perp}^{(m)}} \frac{2 a_{m}}{L} \frac{\sin \left(2 a_{m} z / L+m \pi / 2\right)}{\cos \left(a_{m}+m \pi / 2\right)}\right], \quad m \geqslant m^{(\mathrm{TE})} \\
& \mathbf{E}_{\Omega \mathbf{k}_{\perp}^{(m)}}^{(u \mathrm{TM})}=\frac{-\mathcal{N}_{m}^{(\mathrm{TM})} e^{i \mathbf{k}_{\perp}^{(m)} \cdot \mathbf{x}_{\perp}}}{4 \pi \sqrt{\Omega \sin \kappa_{m}}}\left(e^{\Omega t-i \kappa_{m} / 2}-e^{-\Omega t+i \kappa_{m} / 2}\right) \\
& \times\left[\frac{k_{\perp}^{(m)} \mathbf{n}_{z}}{\varepsilon_{\|}} \frac{\cos \left(2 a_{m} z / L+m \pi / 2\right)}{\cos \left(a_{m}+m \pi / 2\right)}-i \frac{\mathbf{k}_{\perp}^{(m)}}{\varepsilon_{\perp} k_{\perp}^{(m)}} \frac{2 a_{m}}{L} \frac{\sin \left(2 a_{m} z / L+m \pi / 2\right)}{\cos \left(a_{m}+m \pi / 2\right)}\right], \quad 0 \leqslant m \leqslant m^{(\mathrm{TM})} \\
& \mathbf{B}_{\Omega \mathbf{k}_{\perp}^{(m)}}^{(u \mathrm{TM})}=\frac{i \mathcal{N}_{m}^{(\mathrm{TM})} \mu_{\perp} \sqrt{\Omega}\left(\mathbf{n}_{z} \times \mathbf{k}_{\perp}^{(m)}\right)}{4 \pi k_{\perp}^{(m)} \sqrt{\sin \kappa_{m}}} e^{i \mathbf{k}_{\perp}^{(m)} \cdot \mathbf{x}_{\perp}} \frac{\cos \left(2 a_{m} z / L+m \pi / 2\right)}{\cos \left(a_{m}+m \pi / 2\right)}\left(e^{\Omega t-i \kappa_{m} / 2}+e^{-\Omega t+i \kappa_{m} / 2}\right), \quad 0 \leqslant m \leqslant m^{(\mathrm{TM})} .
\end{aligned}
$$

Let us recall that these modes give information about fluctuations and correlations of the electromagnetic field; as long as decoherence does not come into play, the expectation values of the field are null, $\langle\hat{\mathbf{A}}\rangle=\langle\hat{\mathbf{E}}\rangle=\langle\hat{\mathbf{B}}\rangle=\mathbf{0}$. We shall use these modes later, when discussing possible consequences of these analogue instabilities. But first, let us explore more interesting analogies.

\section{SPHERICALLY SYMMETRIC, STATIONARY ANISOTROPIC MEDIUM}

In the previous section, we presented with great amount of detail the canonical quantization scheme for the electromag- netic field in flat space-time in the presence of arbitrary planesymmetric anisotropic polarizable and magnetizable media at linear order. The vacuum of such system was then identified with the vacuum of some nonminimally coupled spin-1 field in a true curved space-time described by the effective metric $g_{\alpha \beta}=\sqrt{n} \operatorname{diag}\left(-n^{-2}, 1,1,1\right)$. The analysis had the advantage of generalizing in a unified language the quantization of various interesting models coming from quantum optics in terms of simple equations (e.g., the Carniglia-Mandel modes [38]). However, the analogue space-time for these configurations is of mathematical interest only and does not capture the symmetry of physical space-times. In order to study more appealing analogues, in this section we turn to spherically 
symmetric configurations, presenting them in a more concise way, for the nuances of the quantization were already explained previously. In this context, we may obtain interesting analogues by also assuming that the medium is able to flow. If the refractive index in a flowing material is high enough, such that the velocity of light becomes smaller than the medium's velocity, then it is clear that a sort of event horizon will form (restricted only to some frequency band which may contain unstable modes). This kind of phenomenon enables us to study analogues of unstable black holes, for instance.

We start working in standard spherical coordinates $(t, r, \theta, \varphi)$, such that $\eta_{\mu \nu}=\operatorname{diag}\left(-1,1, r^{2}, r^{2} \sin ^{2} \theta\right)$. Let the medium's four-velocity field be $v^{\mu}=\gamma(1, v, 0,0)$, where $v=$ $v(r)$ and $\gamma=\left(1-v^{2}\right)^{-1 / 2}$. The effective-metric components then take the form

$g_{\alpha \beta}=\sqrt{n}\left(\begin{array}{cccc}-\gamma^{2}\left(n^{-2}-v^{2}\right) & -\left(1-n^{-2}\right) \gamma^{2} v & 0 & 0 \\ -\left(1-n^{-2}\right) \gamma^{2} v & \gamma^{2}\left(1-n^{-2} v^{2}\right) & 0 & 0 \\ 0 & 0 & r^{2} & 0 \\ 0 & 0 & 0 & r^{2} \sin ^{2} \theta\end{array}\right)$,

where the isotropic parts of the constitutive tensors (in the local, instantaneous rest frame of the medium) are functions of $r[\varepsilon=\varepsilon(r), \mu=\mu(r)]$ and, as usual, $n^{2}=\mu \varepsilon$. As for the traceless anisotropic tensors $\chi_{(\varepsilon)}^{a b}$ and $\chi_{a b}^{(\mu)}$, their components read as

$$
\begin{aligned}
\chi_{(\varepsilon)}^{\alpha \beta}= & \left(\Delta^{(\varepsilon)} / 3\right)\left(2 \gamma^{2} v^{2} \delta_{t}^{\alpha} \delta_{t}^{\beta}+4 \gamma^{2} v \delta_{t}^{(\alpha} \delta_{r}^{\beta)}+2 \gamma^{2} \delta_{r}^{\alpha} \delta_{r}^{\beta}\right. \\
& \left.-\delta_{\theta}^{\alpha} \delta_{\theta}^{\beta} r^{-2}-\delta_{\varphi}^{\alpha} \delta_{\varphi}^{\beta} r^{-2} \sin ^{-2} \theta\right)
\end{aligned}
$$

and

$$
\begin{aligned}
\chi_{\alpha \beta}^{(\mu)}= & \left(\Delta^{(\mu)} / 3\right)\left(2 \gamma^{2} v^{2} \delta_{\alpha}^{t} \delta_{\beta}^{t}-4 \gamma^{2} v \delta_{(\alpha}^{t} \delta_{\beta)}^{r}+2 \gamma^{2} \delta_{\alpha}^{r} \delta_{\beta}^{r}\right. \\
& \left.-\delta_{\alpha}^{\theta} \delta_{\beta}^{\theta} r^{2}-\delta_{\alpha}^{\varphi} \delta_{\beta}^{\varphi} r^{2} \sin ^{2} \theta\right) .
\end{aligned}
$$

Similarly to the plane-symmetric case, these anisotropic tensors simply mean that in the instantaneous local rest frame of the medium, its electric permittivity and magnetic permeability in the radial direction $\left(\varepsilon_{\|}\right.$and $\left.\mu_{\|}\right)$and in the angular directions $\left(\varepsilon_{\perp}\right.$ and $\left.\mu_{\perp}\right)$ satisfy the same relations given below Eqs. (37)-(39): $\varepsilon_{\|}-\varepsilon_{\perp} \equiv \Delta^{(\varepsilon)},\left(2 \varepsilon_{\perp}+\varepsilon_{\|}\right) / 3 \equiv$ $\varepsilon, \mu_{\|}^{-1}-\mu_{\perp}^{-1} \equiv \Delta^{(\mu)}$, and $\left(2 \mu_{\perp}^{-1}+\mu_{\|}^{-1}\right) / 3 \equiv \mu^{-1}$.

Not surprisingly, the laboratory coordinates $(t, r, \theta, \varphi)$ are not the most convenient ones to express Eqs. (18) and (19) in the case of a moving medium. One might initially think that coordinates $(\tau, r, \theta, \varphi)$ which diagonalize the components of the effective metric, obtained by defining $\tau:=t-p(r)$, with $p(r)$ satisfying

$$
\frac{d p}{d r}=-\frac{\left(n^{2}-1\right) v}{1-n^{2} v^{2}},
$$

would lead to the simplest form of the field equations. In these coordinates, the effective line element $d s_{\text {eff }}^{2}$ becomes

$$
d s_{\text {eff }}^{2}=\sqrt{n}\left[-n^{-2} F d \tau^{2}+F^{-1} d r^{2}+r^{2}\left(d \theta^{2}+\sin ^{2} \theta d \varphi^{2}\right)\right],
$$

where $F=\gamma^{2}\left(1-n^{2} v^{2}\right)$. It is noteworthy that for $n=$ constant $>0$ (such that the factors of $n$ in $d s_{\text {eff }}^{2}$ can be absorbed via $\tau \mapsto n^{3 / 4} \tau$ and $r \mapsto n^{-1 / 4} r$ ), then the line element above can be made to represent Schwarzschild space-time by tuning $v$ so that $F \equiv\left(1-r_{s} / r\right)$, where $r_{s}$ is some positive constant. This is achieved by a velocity field satisfying $v^{2}=$ $\left[1+\left(n^{2}-1\right) r / r_{s}\right]^{-1}(n \neq 1)$.

Despite this apparent simplification, the coordinate $\tau=$ $t-p(r)$ with $p$ satisfying Eq. (91) is not convenient to express Maxwell's equations in anisotropic media. This is due to the kinematic polarization (respectively, magnetization) caused by the magnetic (respectively, electric) field. In the case of small velocities and isotropic materials, this effect is modeled by Minkowski's equations [45]. The coordinates $(\tau, r, \theta, \varphi)$ defined using Eq. (91) "diagonalizes" only the isotropic part of the theory and do not take into account the anisotropies. It turns out that a much better choice is obtained by setting $\tau:=t-p(r)$ and replacing condition given in Eq. (91) by

$$
\frac{d p}{d r}=-\frac{\left(n_{\|}^{2}-1\right) v}{1-n_{\|}^{2} v^{2}}
$$

where, again, $n_{\|}^{2}:=\mu_{\perp} \varepsilon_{\perp}$. This choice fully decouples the electromagnetic field modes in the anisotropic, moving material medium, as we shall see below.

Introducing again the four-potential $A_{\mu}$ via $F_{\mu \nu}=\partial_{\mu} A_{\nu}$ $\partial_{\nu} A_{\mu}$, in these new coordinates $(\tau, r, \theta, \varphi)$, the convenient (generalized Coulomb) gauge conditions read as $A_{\tau}=0$ and

$$
\partial_{\varrho}\left(\varepsilon_{\|} r^{2} A_{r}\right)+\partial_{\perp} \cdot \mathbf{A}_{\perp}=0,
$$

where $\varrho$ is merely an auxiliary variable such that $d r / d \varrho \equiv$ $\gamma^{2}\left(1-n_{\|}^{2} v^{2}\right) / \varepsilon_{\perp}, \mathbf{A}_{\perp}=\left(A_{\theta}, A_{\varphi}\right), \partial_{\perp}$ is the derivative operator on the unit sphere compatible with its metric, and it is understood that $r$ is a function of the auxiliary variable $\varrho$. In this gauge, Maxwell's equations lead to

$$
\begin{gathered}
{\left[-\frac{\mu_{\perp}}{\varepsilon_{\perp}} \partial_{\tau}^{2}+\partial_{\varrho}^{2}+\frac{\gamma^{2}\left(1-n_{\|}^{2} v^{2}\right)}{\varepsilon_{\perp} \varepsilon_{\|} r^{2}} \Delta_{S}^{(0)}\right]\left(\varepsilon_{\|} r^{2} A_{r}\right)=0,} \\
{\left[-\frac{\varepsilon_{\perp}}{\mu_{\perp}} \partial_{\tau}^{2}+\partial_{\rho}^{2}+\frac{\gamma^{2}\left(1-n_{\|}^{2} v^{2}\right)}{\mu_{\perp} \mu_{\|} r^{2}}\left(\Delta_{S}^{(1)}-1\right)\right] \mathbf{A}_{\perp}} \\
=\partial_{\perp}\left[\partial_{\rho}\left(\frac{d r}{d \rho} A_{r}\right)-\frac{\mu_{\perp}}{r^{2} \mu_{\|} \varepsilon_{\perp}} \frac{d r}{d \rho} \partial_{\rho}\left(\varepsilon_{\|} r^{2} A_{r}\right)\right]
\end{gathered}
$$

where $\rho$ appearing in Eq. (96) is another auxiliary variable defined through $d r / d \rho \equiv \gamma^{2}\left(1-n_{\|}^{2} v^{2}\right) / \mu_{\perp}$ and $\Delta_{S}^{(0)}$ and $\Delta_{S}^{(1)}$ are the Laplacian operators defined on the unit sphere, acting on scalar and covector fields, respectively.

In order to solve these equations, we proceed in close analogy to the plane-symmetric case. First, let us find solutions with $A_{r}=0$, the transverse electric modes $\mathbf{A}^{(\mathrm{TE})}$. The gauge conditions imply that these solutions can be written as $\mathbf{A}^{(\mathrm{TE})}=\left(0, \partial_{\varphi} \psi / \sin \theta,-\sin \theta \partial_{\theta} \psi\right)$, where $\psi$ is an auxiliary function to be determined. Making use of the stationarity and spherical symmetry of the present scenario, we can look for field modes of the form $\psi=e^{-i \omega \tau} Y_{\ell m}(\theta, \varphi) f_{\omega \ell}^{(\mathrm{TE})}(r)$, where $Y_{\ell m}$ are the scalar spherical harmonics. Substituting this into Eq. (96), $f_{\omega \ell}^{(\mathrm{TE})}$ must satisfy

$$
\left[-\frac{d^{2}}{d \rho^{2}}+\left(\frac{\gamma^{2}\left(1-n_{\|}^{2} v^{2}\right) \ell(\ell+1)}{r^{2} \mu_{\perp} \mu_{\|}}-\frac{\varepsilon_{\perp} \omega^{2}}{\mu_{\perp}}\right)\right] f_{\omega \ell}^{(\mathrm{TE})}=0,
$$


where it is understood that $r$ is a function of the auxiliary variable $\rho$. Notice the similarity between this equation and Eq. (42). In fact, the boundary condition given by Eq. (22) assumes the same form here as it does in the plane-symmetric case:

$$
\left.\left[\overline{f_{\omega \ell}^{(\mathrm{TE})}} \frac{d}{d \rho} f_{\omega^{\prime} \ell}^{(\mathrm{TE})}-f_{\omega^{\prime} \ell}^{(\mathrm{TE})} \frac{d}{d \rho} \overline{f_{\omega \ell}^{(\mathrm{TE})}}\right]\right|_{\dot{\mathcal{I}}}=0 .
$$

This boundary condition ensures that these modes can be orthonormalized according to the sesquilinear form given in Eq. (21), which in this spherically symmetric scenario assumes the form

$$
\begin{aligned}
\left(A, A^{\prime}\right)= & i \int_{\Sigma_{t}} d \Sigma\left\{\varepsilon_{\|} \bar{A}_{r} \partial_{\tau} A_{r}^{\prime}+\frac{\varepsilon_{\perp} \overline{\mathbf{A}}_{\perp} \cdot \partial_{\tau} \mathbf{A}_{\perp}^{\prime}}{\gamma^{2}\left(1-n_{\|}^{2} v^{2}\right)}\right. \\
& \left.+\frac{\gamma^{2}\left(n_{\|}^{2}-1\right) v}{\mu_{\perp}}\left[\overline{\mathbf{A}}_{\perp} \cdot \partial_{r} \mathbf{A}_{\perp}^{\prime}-\left(\overline{\mathbf{A}}_{\perp} \cdot \partial_{\perp}\right) A_{r}^{\prime}\right]\right\} \\
& -\left(\overline{\mathbf{A}} \leftrightarrow \mathbf{A}^{\prime}\right)
\end{aligned}
$$

with $\Sigma_{t}$ being a spacelike surface $t=$ constant. After some tedious but straightforward manipulations (presented in the Appendix), we obtain the final form of normalized, positivefrequency TE modes:

$$
\mathbf{A}_{\omega \ell m}^{(\mathrm{TE})}=\frac{\left(0, i m / \sin \theta,-\sin \theta \partial_{\theta}\right)}{\sqrt{2 \omega \ell(\ell+1)}} e^{-i \omega \tau} Y_{\ell m}(\theta, \varphi) f_{\omega \ell}^{(\mathrm{TE})}(r),
$$

with $f_{\omega \ell}^{(\mathrm{TE})}$ satisfying Eqs. (97) and (98), and normalized according to

$$
\int_{\mathcal{I}_{\varrho}} d \varrho \overline{f_{\omega \ell}^{(\mathrm{TE})}} f_{\omega^{\prime} \ell}^{(\mathrm{TE})}=\delta_{\omega \omega^{\prime}}
$$

Note that the integration variable is $\varrho$ [instead of $\rho$ appearing in Eq. (97)] and $\mathcal{I}_{\varrho}$ stands for the domain of integration in this variable corresponding to $\mathcal{I}$ in coordinate $r$.

Now, let us look for solutions with $A_{r} \not \equiv 0$, the transverse magnetic modes $\mathbf{A}^{(\mathrm{TM})}$. Let $\phi$ be such that $\Delta_{S}^{(0)} \phi=$ $-r^{2} \varepsilon_{\|} A_{r}$. Thus, the gauge conditions lead to $\mathbf{A}^{(\mathrm{TM})}=$ $\left(-r^{-2} \varepsilon_{\|}^{-1} \Delta_{S}^{(0)}, \partial_{\theta} \partial_{\varrho}, \partial_{\varphi} \partial_{\varrho}\right) \phi$. Using again stationarity and spherical symmetry $\phi=e^{-i \omega \tau} Y_{\ell m}(\theta, \varphi) f_{\omega \ell}^{(\mathrm{TM})}(r)$, we obtain that $f_{\omega \ell}^{(\mathrm{TM})}(r)$ satisfies

$$
\left[-\frac{d^{2}}{d \varrho^{2}}+\left(\frac{\gamma^{2}\left(1-n_{\|}^{2} v^{2}\right) \ell(\ell+1)}{r^{2} \varepsilon_{\perp} \varepsilon_{\|}}-\frac{\mu_{\perp} \omega^{2}}{\varepsilon_{\perp}}\right)\right] f_{\omega \ell}^{(\mathrm{TM})}=0 .
$$

Notice, again, the similarity between this equation and Eq. (49). And, again, the boundary condition imposed by Eq. (22) to these modes takes the same form as in the planesymmetric case:

$$
\left.\left[\omega^{2} \overline{f_{\omega \ell}^{(\mathrm{TM})}} \frac{d}{d \varrho} f_{\omega^{\prime} \ell}^{(\mathrm{TM})}-\omega^{\prime 2} f_{\omega^{\prime} \ell}^{(\mathrm{TM})} \frac{d}{d \varrho} \overline{f_{\omega \ell}^{(\mathrm{TM})}}\right]\right|_{\dot{\mathcal{I}}}=0 .
$$

Properly orthonormalizing these modes using Eq. (A1) (see Appendix) leads to the positive-frequency TM normal modes

$$
\begin{aligned}
\mathbf{A}_{\omega \ell m}^{(\mathrm{TM})}= & \frac{\left(r^{-2} \varepsilon_{\|}^{-1} \ell(\ell+1), \partial_{\theta} \partial_{\varrho}, i m \partial_{\varrho}\right)}{\sqrt{2 \omega^{3} \ell(\ell+1)}} \\
& \times e^{-i \omega \tau} Y_{\ell m}(\theta, \varphi) f_{\omega \ell}^{(\mathrm{TM})}(r),
\end{aligned}
$$

with $f_{\omega \ell}^{(\mathrm{TM})}$ satisfying Eqs. (102) and (103), and normalized according to

$$
\int_{\mathcal{I}_{\rho}} d \rho \overline{f_{\omega \ell}^{(\mathrm{TM})}} f_{\omega^{\prime} \ell}^{(\mathrm{TM})}=\delta_{\omega \omega^{\prime}}
$$

Similarly to the TE case, note that the integration variable is not the same which appears in the differential equation (102). $\left(\mathcal{I}_{\rho}\right.$ stands for the domain of integration in the variable $\rho$ corresponding to $\mathcal{I}$ in coordinate $r$.)

The electromagnetic field operator can be represented in terms of the TE and TM modes (and their complex conjugates) as

$$
\hat{\mathbf{A}}=\sum_{\mathrm{J} \in\{\mathrm{TE}, \mathrm{TM}\}} \sum_{\ell m} \int_{\mathcal{E}_{\ell+}^{(\mathrm{J})}} d \omega\left[\hat{a}_{\omega \ell m}^{(\mathrm{J})} \mathbf{A}_{\omega \ell m}^{(\mathrm{J})}+\text { H.c. }\right]
$$

where $\mathcal{E}_{\ell+}^{(\mathrm{J})}:=\mathcal{E}_{\ell}^{(\mathrm{J})} \cap \mathbb{R}_{+}^{*}$, with $\mathcal{E}_{\ell}^{(\mathrm{J})}$ being the set of $\omega$ values for which Eqs. (97) and (98), for J = TE, and Eqs. (102) and (103), for $\mathrm{J}=\mathrm{TM}$, have nontrivial solutions. The orthonormality of TE and TM modes,

$$
\begin{aligned}
\left(A_{\omega \ell m}^{(\mathrm{J})}, A_{\omega^{\prime} \ell^{\prime} m^{\prime}}^{\left(\mathrm{J}^{\prime}\right)}\right. & =-\left(\overline{A_{\omega \ell m}^{(\mathrm{J})}}, \overline{A_{\omega^{\prime} \ell^{\prime} m^{\prime}}^{\left(\mathrm{J}^{\prime}\right)}}\right) \\
& =\delta_{\mathrm{J} \mathrm{J}^{\prime}} \delta_{\omega \omega^{\prime}} \delta_{\ell \ell^{\prime}} \delta_{m m^{\prime}}, \\
\left(A_{\omega \ell m}^{(\mathrm{J})}, \overline{A_{\omega^{\prime} \ell^{\prime} m^{\prime}}^{\left(\mathrm{J}^{\prime}\right)}}\right) & =0,
\end{aligned}
$$

requires that the canonical commutation relations

$$
\begin{aligned}
& {\left[\hat{a}_{\omega \ell m}^{(\mathrm{J})}, \hat{a}_{\omega^{\prime} \ell^{\prime} m^{\prime}}^{\left(\mathrm{J}^{\prime}\right)^{\dagger}}\right]=\delta^{\mathrm{JJ}} \delta_{\omega \omega^{\prime}} \delta_{\ell \ell^{\prime}} \delta_{m m^{\prime}},} \\
& {\left[\hat{a}_{\omega \ell m}^{(\mathrm{J})}, \hat{a}_{\omega^{\prime} \ell^{\prime} m^{\prime}}^{\left(\mathrm{J}^{\prime}\right)}\right]=0}
\end{aligned}
$$

hold.

\section{A. Instability analysis}

The close similarity between Eqs. (42) and (97) and between Eqs. (49) and (102) makes the instability analysis in this spherically symmetric scenario essentially identical to the one performed in the plane-symmetric case, with $\ell(\ell+1)$ playing the role $k_{\perp}^{2}$ did in Eq. (58). So, putting the effective potentials of Eqs. (97) and (102), with $\omega^{2}=-\Omega^{2}$, in the form

$$
V_{\mathrm{eff}}=C_{1} \ell(\ell+1)+C_{2} \Omega^{2}
$$

we again have two types of instabilities: (i) large- $\ell$ instability, when $C_{1}<0$ somewhere, and (ii) minimum-thickness instability, when $C_{1}>0$ but $C_{2}<0$ in a sufficiently thick spherical shell [see discussion below Eq. (58)]. The only additional feature is that, by allowing the medium to flow, type-(i) (large$\ell$ ) instability for both TE and TM modes can arise when the medium's velocity $v(r)$ exceeds the radial light velocity $n_{\|}^{-1}$.

Let $g_{\Omega \ell}^{(\mathrm{J})}$ represent the solutions of Eqs. (97) (for $\mathrm{J}=\mathrm{TE}$ ) and (102) (for $\mathbf{J}=\mathrm{TM}$ ), subject to the boundary conditions given by Eqs. (98) and (103), respectively, with $\omega^{2}=-\Omega^{2}$ ( $\Omega>0$, without loss of generality). The normalized, unstable modes are presented below (see Appendix for details). 


\section{Unstable TE modes}

Unstable TE modes orthonormalized according to the analogou of Eqs. (107) and (108) read as (up to global phase and time translation)

$$
\begin{aligned}
\mathbf{A}_{\Omega \ell m}^{(u \mathrm{TE})}= & \frac{\left(e^{\Omega \tau-i s_{\varepsilon}^{\perp} \kappa / 2}+e^{-\Omega \tau+i s_{\varepsilon}^{\perp} \kappa / 2}\right)}{2 \sqrt{\Omega \ell(\ell+1) \sin \kappa}} g_{\Omega \ell}^{(\mathrm{TE})}(r) \\
& \times\left(0, i m / \sin \theta,-\sin \theta \partial_{\theta}\right) Y_{\ell m}(\theta, \varphi),
\end{aligned}
$$

with $\kappa$ being a constant $(0<\kappa<\pi), g_{\Omega \ell}^{(\mathrm{TE})}$ normalized according to

$$
\left|\int_{\mathcal{I}} d r \frac{\varepsilon_{\perp}}{\gamma^{2}\left(1-n_{\|}^{2} v^{2}\right)} \overline{g_{\Omega \ell}^{(\mathrm{TE})}}(r) g_{\Omega^{\prime} \ell}^{(\mathrm{TE})}(r)\right|=\delta_{\Omega \Omega^{\prime}},
$$

and $s_{\varepsilon}^{\perp}$ being the sign of the integral above. Calculating the electric $\mathbf{E}_{\Omega \ell m}^{(u \mathrm{TE})}$ and magnetic $\mathbf{B}_{\Omega \ell m}^{(u \mathrm{TE})}$ vector fields associated to these modes in the laboratory frame, we have

$$
\begin{aligned}
& \mathbf{E}_{\Omega \ell m}^{(u \mathrm{TE})}=\frac{\sqrt{\Omega}\left(-i m \mathbf{e}_{\theta} / \sin \theta+\mathbf{e}_{\varphi} \partial_{\theta}\right)}{2 r \sqrt{\ell(\ell+1) \sin \kappa}} g_{\Omega \ell}^{(\mathrm{TE})}(r) Y_{\ell m}(\theta, \varphi)\left(e^{\Omega \tau-i s_{\varepsilon}^{\perp} \kappa / 2}-e^{-\Omega \tau+i s_{\varepsilon}^{\perp} \kappa / 2}\right), \\
& \mathbf{B}_{\Omega \ell m}^{(u \mathrm{TE})}=\frac{\left[\ell(\ell+1) \mathbf{e}_{r}+\left(i m \mathbf{e}_{\varphi} / \sin \theta+\mathbf{e}_{\theta} \partial_{\theta}\right) r \partial_{r}\right]}{2 r^{2} \sqrt{\Omega \ell(\ell+1) \sin \kappa}} g_{\Omega \ell}^{(\mathrm{TE})}(r) Y_{\ell m}(\theta, \varphi)\left(e^{\Omega \tau-i s_{\varepsilon}^{\perp} \kappa / 2}+e^{-\Omega \tau+i s_{\varepsilon}^{\perp} \kappa / 2}\right) .
\end{aligned}
$$

\section{Unstable TM modes}

Finally, the unstable TM modes orthonormalized according to the analogues of Eqs. (107) and (108) read as (up to global phase and time translation)

$$
\begin{aligned}
\mathbf{A}_{\Omega \ell m}^{(u \mathrm{TM})}= & \frac{\left(r^{-2} \varepsilon_{\|}^{-1} \ell(\ell+1), \partial_{\theta} \partial_{\varrho}, i m \partial_{\varrho}\right)}{2 \sqrt{\Omega^{3} \ell(\ell+1) \sin \kappa}} g_{\Omega \ell}^{(\mathrm{TM})}(r) \\
& \times Y_{\ell m}(\theta, \varphi)\left(e^{\Omega \tau+i s_{\mu}^{\perp} \kappa / 2}+e^{-\Omega \tau-i s_{\mu}^{\perp} \kappa / 2}\right),
\end{aligned}
$$

with, again, $\kappa$ being a constant $(0<\kappa<\pi), g_{\Omega \ell}^{(\mathrm{TM})}$ normalized according to

$$
\left|\int_{\mathcal{I}} d r \frac{\mu_{\perp}}{\gamma^{2}\left(1-n_{\|}^{2} v^{2}\right)} g_{\Omega \ell}^{(\mathrm{TM})}(r) g_{\Omega^{\prime} \ell}^{(\mathrm{TM})}(r)\right|=\delta_{\Omega \Omega^{\prime}},
$$

and $s_{\mu}^{\perp}$ being the sign of the integral above. Calculating the electric $\mathbf{E}_{\Omega \ell m}^{(u \mathrm{TM})}$ and magnetic $\mathbf{B}_{\Omega \ell m}^{(u \mathrm{TM})}$ vector fields associated to these modes in the laboratory frame, we have

$$
\begin{aligned}
& \mathbf{E}_{\Omega \ell m}^{(u \mathrm{TM})}=-\frac{\left[\ell(\ell+1) \mathbf{e}_{r} / \varepsilon_{\|}+\left(i m \mathbf{e}_{\varphi} / \sin \theta+\mathbf{e}_{\theta} \partial_{\theta}\right) r \partial_{\varrho}\right]}{2 r^{2} \sqrt{\Omega \ell(\ell+1) \sin \kappa}} g_{\Omega \ell}^{(\mathrm{TM})}(r) Y_{\ell m}(\theta, \varphi)\left(e^{\Omega \tau+i s_{\mu}^{\perp} \kappa / 2}-e^{-\Omega \tau-i s_{\mu}^{\perp} \kappa / 2}\right), \\
& \mathbf{B}_{\Omega \ell m}^{(u \mathrm{TM})}=\frac{\mu_{\perp} \sqrt{\Omega}\left(-i m \mathbf{e}_{\theta} / \sin \theta+\mathbf{e}_{\varphi} \partial_{\theta}\right)}{2 r \gamma^{2}\left(1-n_{\|}^{2} v^{2}\right) \sqrt{\ell(\ell+1) \sin \kappa}} g_{\Omega \ell}^{(\mathrm{TM})}(r) Y_{\ell m}(\theta, \varphi)\left(e^{\Omega \tau+i s_{\mu}^{\perp} \kappa / 2}+e^{-\Omega \tau-i s_{\mu}^{\perp} \kappa / 2}\right) .
\end{aligned}
$$

As argued in the previous case, when instability is triggered and modes $\mathbf{A}_{\Omega \ell m}^{(u \mathrm{~J})}$ appear, they must be included in the field expansion given by Eq. (106), along with their complex conjugates. Eventually $\left(t \gg \Omega^{-1}\right.$ ), these modes dominate the field fluctuations.

\section{B. Example}

Now, let us consider a concrete scenario where electromagnetism in a gravitationally interesting system, nonminimally coupled to the background geometry via $\chi^{\text {abcd }}$ given by Eq. (23) (but with arbitrary $\alpha_{1}, \alpha_{2}, \alpha_{3}$ ), can be mimicked by an anisotropic, stationary moving medium. We have already seen that setting $n=$ constant and $v^{2}=\left[1+\left(n^{2}-1\right) r / r_{s}\right]^{-1}$ leads to an effective line element which describes the vacuum Schwarzschild space-time. In this case, Eq. (29) is trivially satisfied and Eqs. (26)-(28) give

$$
\begin{aligned}
\mu & =n, \\
\Delta^{(\varepsilon)} & =3 \alpha_{1} n^{1 / 2} \frac{r_{s}}{r^{3}}, \\
\Delta^{(\mu)} & =\frac{3 \alpha_{1}}{n^{3 / 2}} \frac{r_{s}}{r^{3}},
\end{aligned}
$$

which lead to the material properties

$$
\begin{aligned}
\varepsilon_{\perp} & =n\left(1-\frac{\alpha_{1} r_{s}}{n^{1 / 2} r^{3}}\right), \\
\varepsilon_{\|} & =n\left(1+\frac{2 \alpha_{1} r_{s}}{n^{1 / 2} r^{3}}\right), \\
\mu_{\perp} & =n\left(1-\frac{\alpha_{1} r_{s}}{n^{1 / 2} r^{3}}\right)^{-1}, \\
\mu_{\|} & =n\left(1+\frac{2 \alpha_{1} r_{s}}{n^{1 / 2} r^{3}}\right)^{-1} .
\end{aligned}
$$

We promptly see that $n_{\|}:=\sqrt{\mu_{\perp} \varepsilon_{\perp}}=n$, which shows that the analogue horizon for these nonminimally coupled modes, located where $v^{2}=n_{\|}^{-2}$, coincides with the analogue Schwarzschild radius $r_{s}$. [Note, however, that this system is analogous to a physical black hole with Schwarzschild radius $R_{s}=n^{1 / 4} r_{s}$, due to absorption of $\sqrt{n}$ in Eq. (92).] As for the other refractive indices $n_{\perp}^{\text {(TE) }}:=\sqrt{\mu_{\|} \varepsilon_{\perp}}$ and $n_{\perp}^{(\mathrm{TM})}:=$ $\sqrt{\mu_{\perp} \varepsilon_{\|}}\left(=n^{2} / n_{\perp}^{(\mathrm{TE})}\right)$, Fig. 3 shows their squared values (in black and red, respectively) for positive (solid lines) and negative (dashed lines) values of $\alpha_{1}$. Note that, depending on the values of $\alpha_{1} /\left(n^{1 / 2} r_{s}^{2}\right)$, some kind of metamaterial (possibly with some negative squared refractive indices) may be needed in order to mimic this nonminimal coupling of the 


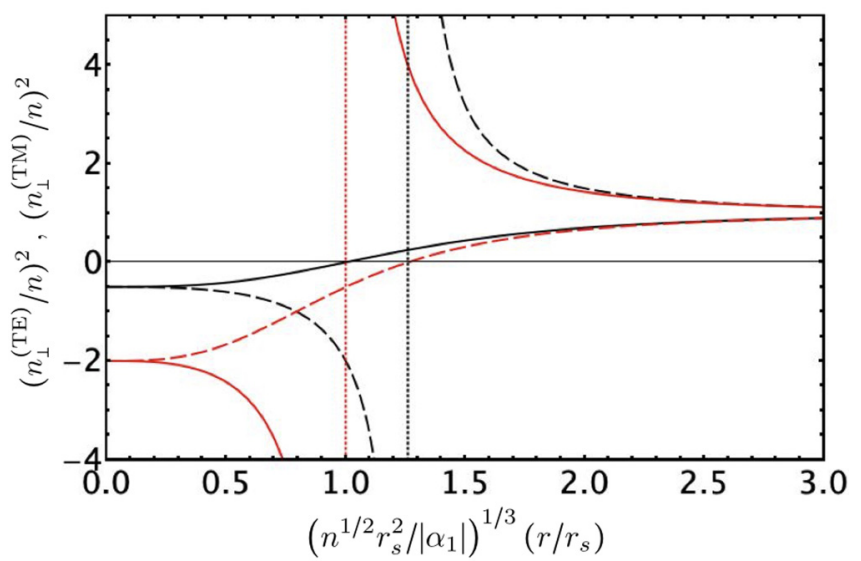

FIG. 3. Squared values of the refractive indices $n_{\perp}^{(\mathrm{TE})}$ (in black) and $n_{\perp}^{\text {(TM) }}$ (in red) for positive (solid lines) and negative (dashed lines) values of $\alpha_{1}$. The black and red dotted lines mark where $n_{\perp}^{\text {(TE) }}$ (for negative $\alpha_{1}$ ) and $n_{\perp}^{(\mathrm{TM})}$ (for positive $\alpha_{1}$ ) are singular, respectively.

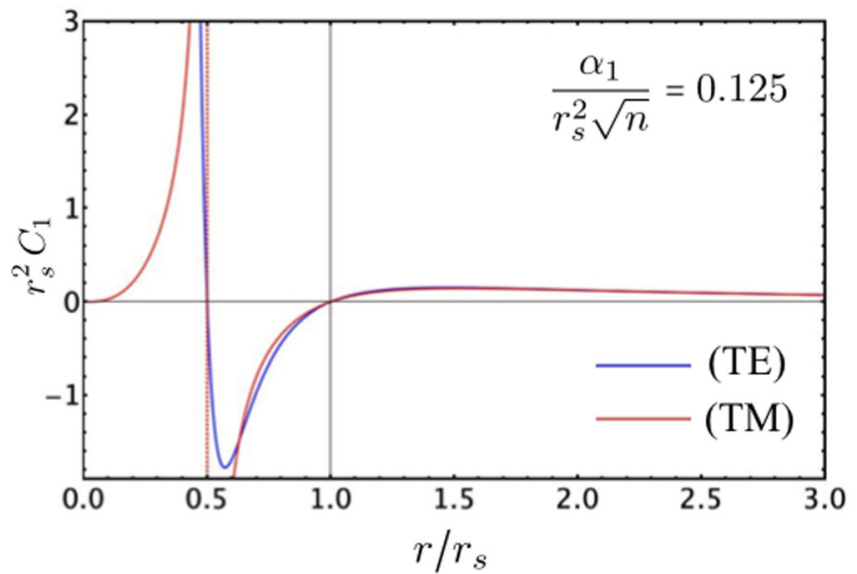

(a)

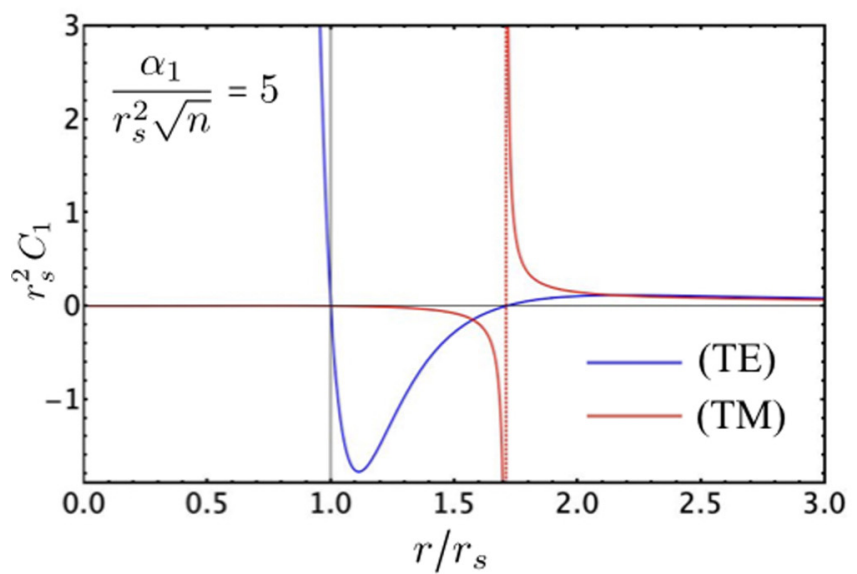

(b) electromagnetic field with the Riemann curvature tensor in the exterior region of a Schwarzschild black hole. Conversely, regardless how difficult it may be to set up such an experimental configuration in the laboratory, it is interesting in its own that QED-inspired nonminimally coupled electromagnetism in the background of a black hole behaves as in such an exotic metamaterial in flat space-time.

Turning to the question of possible instabilities, in Fig. 4 we show the behavior of the terms $C_{1}$ and $C_{2}$ appearing in Eq. (111) for the TE (in blue) and TM (in red) modes extracted, respectively, from Eqs. (97) and (102):

$$
\begin{aligned}
& C_{1}=\left\{\begin{array}{l}
n^{-2} r^{-9}\left(r-r_{s}\right)\left(r^{3}-\frac{\alpha_{1} r_{s}}{\sqrt{n}}\right)\left(r^{3}+\frac{2 \alpha_{1} r_{s}}{\sqrt{n}}\right), \\
\frac{n^{-2}\left(r-r_{s}\right) r^{3}}{\left(r^{3}-\alpha_{1} r_{s} / \sqrt{n}\right)\left(r^{3}+2 \alpha_{1} r_{s} / \sqrt{n}\right)},
\end{array}\right. \\
& C_{2}=\left\{\begin{array}{l}
\left(1-\frac{\alpha_{1} r_{s}}{r^{3} \sqrt{n}}\right)^{2}, \\
\left(1-\frac{\alpha_{1} r_{s}}{r^{3} \sqrt{n}}\right)^{-2},
\end{array}\right.
\end{aligned}
$$

where the first and second lines in the expressions above refer to the TE and TM modes, respectively. Figure 4(a) is

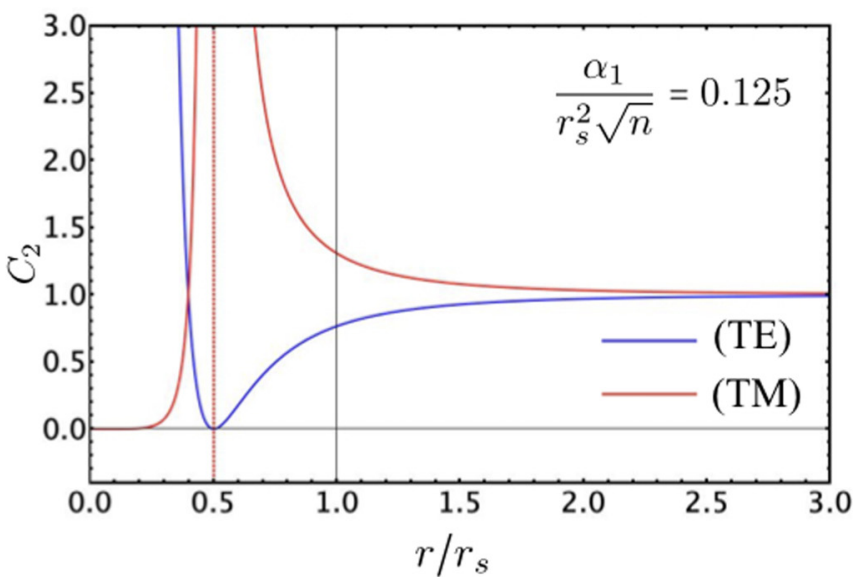

(c)

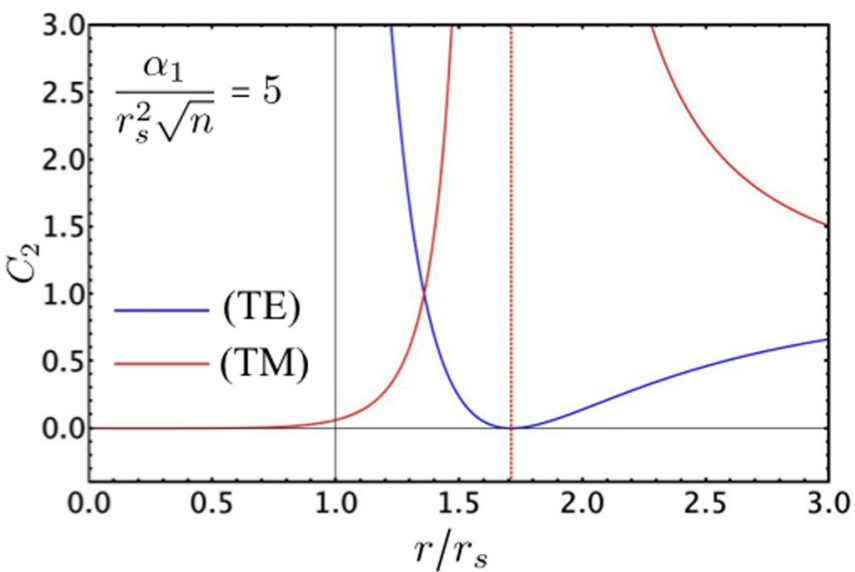

(d)

FIG. 4. Plot of the coefficients $C_{1}\left[(\mathrm{a})\right.$ and (b)] and $C_{2}$ [(c) and (d)] appearing in Eq. (111) for electromagnetic modes TE (blue curves) and TM (red curves), nonminimally coupled to the background geometry of a Schwarzschild black hole via Eq. (23). (a), (c) Illustrate the general behavior of $C_{1}$ and $C_{2}$ for $-r_{s}^{2} \sqrt{n} / 2<\alpha_{1}<r_{s}^{2} \sqrt{n}$, while (b), (d) are representative of the behavior of $C_{1}$ and $C_{2}$ for $\alpha_{1}<-r_{s}^{2} \sqrt{n} / 2$ or $\alpha_{1}>r_{s}^{2} \sqrt{n}$. According to the instability discussion, only large- $\ell$ instability can appear in this case since $C_{2} \geqslant 0$ everywhere. Moreover, for $\alpha_{1}<-r_{s}^{2} \sqrt{n} / 2$ or $\alpha_{1}>r_{s}^{2} \sqrt{n}$, the unstable modes can be mostly supported outside the analogous event horizon $r>r_{s}$. 
representative of the behavior of $C_{1}$ for $-r_{s}^{2} \sqrt{n} / 2<\alpha_{1}<$ $r_{s}^{2} \sqrt{n}$, while Fig. 4(b) gives the correct qualitative behavior of $C_{1}$ for $\alpha_{1}<-r_{s}^{2} \sqrt{n} / 2$ or $\alpha_{1}>r_{s}^{2} \sqrt{n}$. Figures 4(c) and 4(d) show the behavior of $C_{2}$ for the same values of $\alpha_{1}$ used in Figs. 4(a) and 4(b).

It is clear, from the expressions above, that $C_{2}$ is everywhere non-negative, while $C_{1}$ assumes negative values in the region with radial coordinate $r$ between $\left(\alpha_{1} r_{s} / \sqrt{n}\right)^{1 / 3}$ and $r_{s}$ (if $\alpha_{1}>0$ ) or between $\left[\left|\alpha_{1}\right| r_{s} /(2 \sqrt{n})\right]^{1 / 3}$ and $r_{s}$ (if $\alpha_{1}<0$ ). Therefore, according to the discussion of Sec. IV A, this nonminimally coupled electromagnetic theory in Schwarzschild space-time exhibits large- $\ell$ instability. In particular, if $\alpha_{1}>$ $r_{s}^{2} \sqrt{n}$ or $\alpha_{1}<-2 r_{s}^{2} \sqrt{n}$, then the unstable modes influence the exterior region of the back hole.

\section{STABILIZATION: SPONTANEOUS VECTORIZATION, PHOTO PRODUCTION, AND LONG-RANGE INDUCED CORRELATIONS}

We now turn our attention to discussing what can possibly happen to the analogous system when the vacuum instability is triggered. In the gravitational scenario, it has been shown that in some cases (for instance, depending on the fieldbackground coupling), stabilization occurs due to the appearance of a nonzero value for the field (spontaneous scalarization or vectorization) [14-18], seeded by decoherence of the growing initial-vacuum fluctuations [13]. In this process, field particles and waves are produced [14,28] and carry away the energy excess of the initial vacuum state in comparison to the stabilized configuration.

If we transpose these conclusions, mutatis mutandis, to our analogous systems, then an electromagnetic field should spontaneously appear in the material, bringing the whole system to a new equilibrium configuration through nonlinear effects brought in by field-dependent constitutive tensors $\varepsilon^{a b}$ and $\mu_{a b}$ [see Eqs. (7) and (8)], with photons being emitted, carrying away the energy excess. Although the detailed dynamics of the stabilization processes in the gravitational and in the analogous systems are quite different (ruled by Einstein equations in the gravitational case and by the macroscopic Maxwell's equations with field-dependent $\varepsilon^{a b}$ and $\mu_{a b}$ in the analogous systems), the qualitative features of the whole process, described above, seem quite reasonable to occur in generic field stabilization processes.

It is important to mention that the timescale set by the instability $\Omega^{-1}$ is typically of the order of the time light takes to travel the typical size of the system, $L$. Therefore, in the analogous laboratory scenarios, the stabilization process would occur almost instantaneously $\left[\sim L /(1 \mathrm{~cm}) \times 10^{-10} \mathrm{~s}\right]$ once the instability conditions are met, which, for a given system, may depend on external parameters such as temperature, external fields, etc., through their influence on the constitutive functions $\varepsilon_{\perp}, \varepsilon_{\|}, \mu_{\perp}, \mu_{\|}$. The whole process would most likely be interpreted as a kind of phase transition, where the "longrange" emergent correlations in the material would come from interaction of its constituents with a common (initially unstable vacuum) fluctuating mode and/or the stabilized field configuration.

For concreteness sake, let us consider the explicit form of the unstable modes found in the example of Sec. III, where instability occurs due to a negative value of $\mu_{\perp}$, for some (isolated) $\omega^{2}=-\Omega^{2}<0$, in a homogeneous slab of width $L$. Although this system is not analogous to vacuum nonminimally coupled electromagnetism in any realistic space-time, it serves to illustrate general features of the mechanism itself, in addition to being much simpler to set up in the laboratory. This is no different than looking for fingerprints of analogue Hawking radiation in systems whose only similarity with realistic black holes is the presence of an effective event horizon, which is the common approach in condensed-matter and optical experimental analogues.

As argued before, once instability sets in, the unstable modes must be added to the expansion of the field operator $\hat{\mathbf{A}}$, along with their complex conjugates, with corresponding annihilation $\hat{a}_{\Omega \mathbf{k}}^{(u \mathrm{~J})}$ and creation $\hat{a}_{\Omega \mathbf{k}}^{(u \mathrm{~J}) \dagger}$ operators. It is easy to see that the field's vacuum fluctuations and correlations are eventually $\left(t, t^{\prime} \gg \Omega^{-1}\right)$ dominated by these unstable modes, at least as long as decoherence does not come into play. The dominant contribution to the vacuum correlations in the example of Sec. III B reads as (the reader should refer to Sec. III B for the definition of all quantities appearing in these expressions)

$$
\begin{aligned}
\left\langle A_{j}(x) A_{l}\left(x^{\prime}\right)\right\rangle & \sim \frac{2 \pi}{L_{\perp}} \int_{0}^{2 \pi} d \varphi\left\{\sum_{m=0}^{m^{\mathrm{TM})}} k_{\perp}^{(m)}\left[\mathbf{A}_{\Omega \mathbf{k}_{\perp}^{(m)}}^{(\mathrm{uTM})}(x)\right]_{j}\left[\overline{\mathbf{A}_{\Omega \mathbf{k}_{\perp}^{(m)}}^{(\mathrm{uTM})}}\left(x^{\prime}\right)\right]_{l}+\sum_{m=m^{(\mathrm{TE})}}^{\infty} k_{\perp}^{(m)}\left[\mathbf{A}_{\Omega \mathbf{k}_{\perp}^{(m)}}^{(\mathrm{uTE})}(x)\right]_{j}\left[\overline{\mathbf{A}_{\Omega \mathbf{k}_{\perp}^{(m)}}^{(\mathrm{uTT})}}\left(x^{\prime}\right)\right]_{l}\right\} \\
& \sim \frac{e^{\Omega\left(t+t^{\prime}\right)}}{4 L_{\perp} \Omega}\left\{\sum_{m=0}^{m^{(\mathrm{TM})}} \frac{k_{\perp}^{(m)}}{\sin \kappa_{m}} \mathcal{D}_{j l(m)}^{(\mathrm{TM})}\left(d_{\perp}\right) g_{\Omega k_{\perp}^{(m)}}^{(\mathrm{TM})}(z) \overline{g_{\Omega k_{\perp}^{(m)}}^{(\mathrm{TM})}}\left(z^{\prime}\right)+\sum_{m=m^{(\mathrm{TE})}}^{\infty} \frac{k_{\perp}^{(m)}}{\sin \kappa_{m}} \mathcal{D}_{j l(m)}^{(\mathrm{TE})}\left(d_{\perp}\right) g_{\Omega k_{\perp}^{(m)}}^{(\mathrm{TE})}(z) \overline{g_{\Omega k_{\perp}^{(m)}}^{(\mathrm{TE})}}\left(z^{\prime}\right)\right\},
\end{aligned}
$$

where $\varphi$ is the angle between $\mathbf{k}_{\perp}$ and $\left(\mathbf{x}_{\perp}-\mathbf{x}_{\perp}^{\prime}\right), d_{\perp}:=\left\|\mathbf{x}_{\perp}-\mathbf{x}_{\perp}^{\prime}\right\|$, and the operators $\mathcal{D}_{j l(m)}^{(\mathrm{J})}\left(d_{\perp}\right)$ acting on $g_{\Omega k_{\perp}^{(m)}}^{(\mathrm{J})}(z) \overline{g_{\Omega k_{\perp}^{(m)}}^{(\mathrm{J})}}\left(z^{\prime}\right)$ are defined by

$$
\mathcal{D}_{j l(m)}^{(\mathrm{TM})}\left(d_{\perp}\right):=\frac{1}{\Omega^{2} \varepsilon_{\perp}^{2}}\left[J_{1}^{\prime}\left(k_{\perp}^{(m)} d_{\perp}\right) \delta_{j}^{\ell} \delta_{l}^{\ell}+\frac{J_{1}\left(k_{\perp}^{(m)} d_{\perp}\right)}{k_{\perp}^{(m)} d_{\perp}} \delta_{j}^{\varphi} \delta_{l}^{\varphi}\right] \frac{d^{2}}{d z d z^{\prime}}+\frac{\left(k_{\perp}^{(m)}\right)^{2} J_{0}\left(k_{\perp}^{(m)} d_{\perp}\right)}{\Omega^{2} \varepsilon_{\|}^{2}} \delta_{j}^{z} \delta_{l}^{z}-\frac{k_{\perp}^{(m)} J_{1}\left(k_{\perp}^{(m)} d_{\perp}\right)}{\Omega^{2} \varepsilon_{\|} \varepsilon_{\perp}}\left(\delta_{j}^{\ell} \delta_{l}^{z} \frac{d}{d z}-\delta_{j}^{z} \delta_{l}^{\ell} \frac{d}{d z^{\prime}}\right),
$$

$$
\mathcal{D}_{j l(m)}^{(\mathrm{TE})}\left(d_{\perp}\right):=\frac{J_{1}\left(k_{\perp}^{(m)} d_{\perp}\right)}{k_{\perp}^{(m)} d_{\perp}} \delta_{j}^{\ell} \delta_{l}^{\ell}+J_{1}^{\prime}\left(k_{\perp}^{(m)} d_{\perp}\right) \delta_{j}^{\varphi} \delta_{l}^{\varphi},
$$




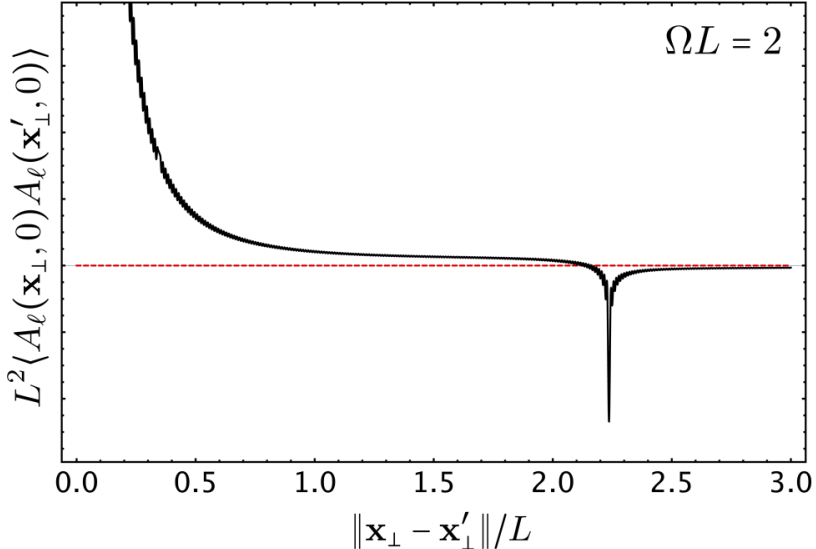

(a)

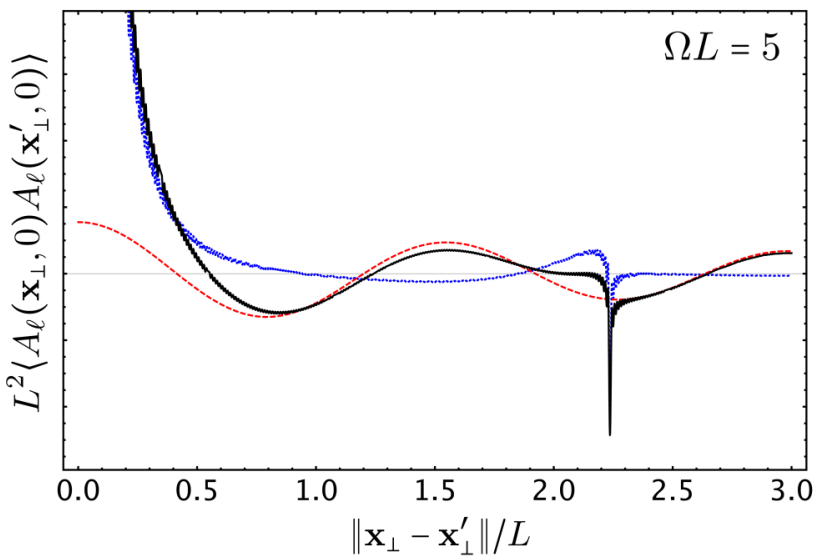

(b)

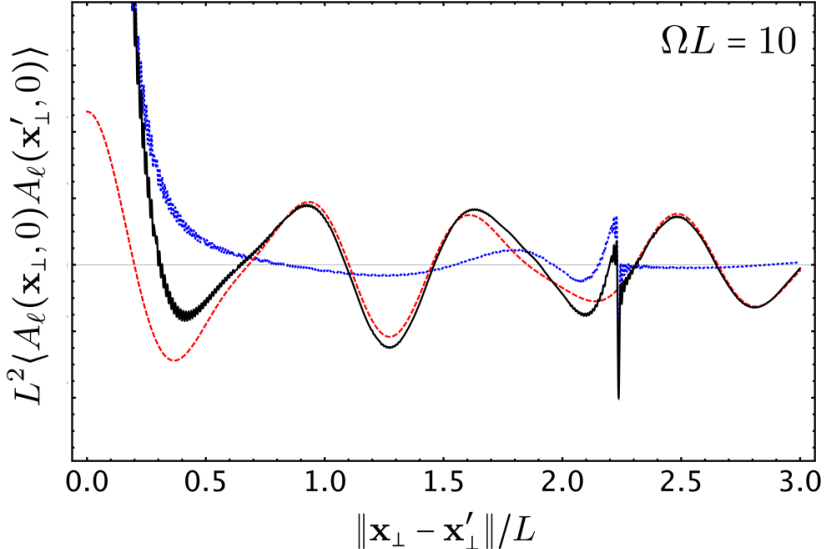

(c)

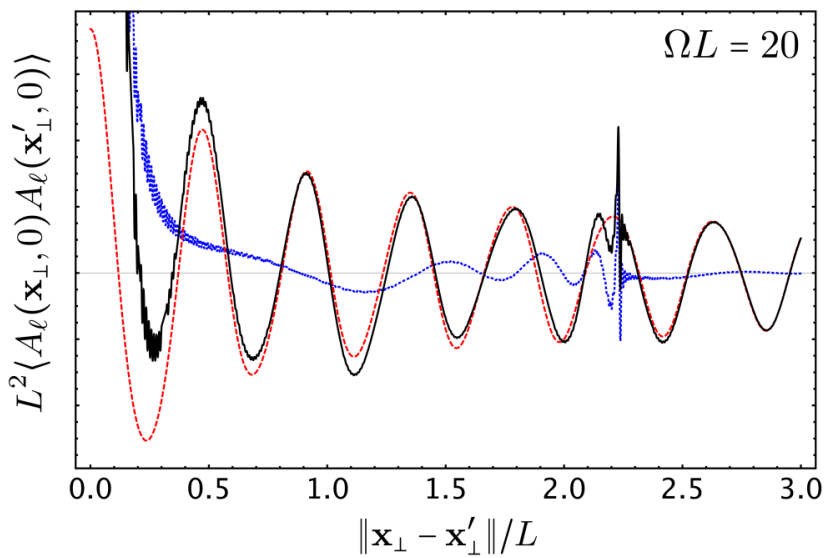

(d)

FIG. 5. Equal-time $\left(t=t^{\prime} \gg \Omega^{-1}\right)$, two-point correlation function $\left\langle A_{\ell}(\mathbf{x}) A_{\ell}\left(\mathbf{x}^{\prime}\right)\right\rangle$ of the component of the quantum field $\hat{\mathbf{A}}$ along the vector separation $\mathbf{x}_{\perp}-\mathbf{x}_{\perp}^{\prime}$, for points in the $z=0$ plane, for different values of $\Omega L$, with same values of constitutive functions given in Fig. 2 . The dotted (blue) lines represent the contribution coming from the TE modes, while the dashed (red) lines depict the contribution coming from the TM modes. The solid (black) lines give the sum of both contributions. Notice that long-range $\left(\left\|\mathbf{x}_{\perp}-\mathbf{x}_{\perp}^{\prime}\right\| \gtrsim L\right)$ correlations are mainly due to the TM modes, which undergo minimum-width instability.

with indices $\ell$ and $\varphi$ standing for vector components along $\left(\mathbf{x}_{\perp}-\mathbf{x}_{\perp}^{\prime}\right)$ and $\mathbf{n}_{z} \times\left(\mathbf{x}_{\perp}-\mathbf{x}_{\perp}^{\prime}\right)$, respectively; $J_{n}$ and $J_{n}^{\prime}$ stand for the Bessel functions of first kind and their first derivatives, respectively. Field correlations $\left\langle E_{j}(x) E_{l}\left(x^{\prime}\right)\right\rangle$ and $\left\langle B_{j}(x) B_{l}\left(x^{\prime}\right)\right\rangle$ can be similarly obtained, in particular, $\left\langle E_{j}(x) E_{l}\left(x^{\prime}\right)\right\rangle \sim \Omega^{2}\left\langle A_{j}(x) A_{l}\left(x^{\prime}\right)\right\rangle$. As an illustration, in Fig. 5 we plot the equal-time $\left(t=t^{\prime} \gg \Omega^{-1}\right)$, longitudinal correlation function $\left\langle A_{\ell}(\mathbf{x}) A_{\ell}\left(\mathbf{x}^{\prime}\right)\right\rangle$ for points $\mathbf{x}, \mathbf{x}^{\prime}$ in the plane $z=0$, for the same values of constitutive functions used in Fig. 2 and four different values of $\Omega L$. The vertical-axis scale is arbitrary, but the same in all plots, since the correlations grow exponentially in time, from their typical (stable-vacuum) values of or$\operatorname{der} \hbar /\left(c L d_{\perp}\right) \sim\left[1 \mathrm{~cm}^{2} /\left(L d_{\perp}\right)\right] \times 10^{-8} \mathrm{eV} /\left(\mathrm{cm}^{3} \mathrm{GHz}^{2}\right)$, until decoherence and vectorization take over. Notice that once minimum-width $(\mathrm{TM})$ instability sets in, macroscopic $(\sim L)$ field correlations are enhanced. It is an interesting question whether any such "long-range" correlation would survive or leave an imprint in the final stable configuration. Although not directly relevant for the analogy with gravity-induced instability itself, such correlations might lead to interesting material behavior.

\section{FINAL REMARKS}

We have shown that gravity-induced instabilities, related to the vacuum-awakening effect in the quantum context [9-11,28] and spontaneous scalarization or vectorization in the classical one [14-18], can be mimicked by electromagnetism in anisotropic metamaterials with appropriate constitutive functions. This follows from the formal analogy between electromagnetism in anisotropic media and nonminimally coupled electromagnetism in curved space-times, presented in Sec. II. We explored two concrete scenarios: (i) a planesymmetric, static slab, whose main interest is its simplicity regarding experimental setup (see Sec. III), and (ii) a spherically symmetric, moving media, whose main feature is its analogy with QED-inspired nonminimally coupled electromagnetism in Schwarzschild space-time [33,34] for given velocity and constitutive-functions profiles (see Secs. II A and IV).

Once instability is triggered in the analogous systems, some stabilization process must take place, leading the system to a new stable configuration. The details of this stabilization process and of the final configuration will most likely depend on specific nonlinear properties of the metamaterial, but it 
seems reasonable that they might involve the appearance of nonzero electromagnetic fields in the material (analogous to spontaneous vectorization in curved space-times) and photo production which carries away the energy excess with respect to the stable configuration. As discussed earlier, the timescale involved in the stabilization process can be very short $\left(\sim 10^{-10} \mathrm{~s}\right)$, which would make it very difficult to even identify the unstable phase. This is similar to what might occur with negative conductivity, which has never been directly measured but which is predicted to lead to zerodc-resistance states [46] which were observed in laboratory $[47,48]$, although an alternative explanation has been proposed [49].

Clearly, the feasibility of such analogues is bound to the existence of material configurations with the required constitutive functions. As briefly pointed out in the Introduction, this can be achieved at least for anisotropic neutral plasmas, and the recent advances in metamaterial science offer a plethora of possible candidates, especially the hyperbolic metamaterials $[42,43]$, that possess precisely the form given in Eqs. (9) and (10) with the required "negativeness." In particular, we call attention to the increase in the spontaneous light emission in such configurations, which may be related to the process of stabilization in active scenarios.

It is also important to mention that the QED-inspired analogues (Sec. II A) are not restricted to the study of vacuum instability. For instance, they can be used to study light ray propagation in the corresponding space-times and one possible application is the QED-induced birefringence in the Schwarzschild space-time [33]. For this particular experiment, one can work far from the effective horizon, where the constitutive coefficients (123)-(126) are positive.

Our main purpose here was to lay down an unexplored class of analogue models of curved-space-time phenomena, with main interest on the gravitational side of the analogy. Notwithstanding, the consequences of the analogue gravityinduced instability to the metamaterial side may be interesting on its own. The electromagnetic field instability may mark, lead, or mediate some kind of phase transition in the metamaterial, where the spontaneously created field and/or its amplified "long-range" correlations may play some important role (see discussion in Sec. V). Investigations along these lines are currently in course and will be presented elsewhere.

\section{ACKNOWLEDGMENT}

C.C.H.R. was mainly supported by São Paulo Research Foundation (FAPESP), through Grant No. 2015/26438-8, and in part by the Coordenação de Aperfeiçoamento de Pessoal de Nível Superior-Brasil (CAPES)-Finance Code 001.

\section{APPENDIX: NORMALIZATION OF STABLE AND UNSTABLE MODES IN THE SPHERICALLY SYMMETRIC CASE}

Here, we present in detail the calculations involved in normalizing the electromagnetic modes in the spherically symmetric case. Since we are dealing with analogues to which there is a natural physical notion of time, the laboratory-frame time $t$, it is convenient to use $t=$ constant surfaces $\left(\Sigma_{t}\right)$ to normalize the modes. Obviously, this choice bears no physical consequence on our results.

The sesquilinear form given in Eq. (21), applied to the scenario described in Sec. IV, takes the form (notice that the integrand is a scalar and, as such, can be evaluated in any coordinate system)

$$
\left(A, A^{\prime}\right)=i \int_{\Sigma_{t}} d \Sigma\left\{\varepsilon_{\|} \bar{A}_{r} \partial_{\tau} A_{r}^{\prime}+\frac{\varepsilon_{\perp} \overline{\mathbf{A}}_{\perp} \cdot \partial_{\tau} \mathbf{A}_{\perp}^{\prime}}{\gamma^{2}\left(1-n_{\|}^{2} v^{2}\right)}+\frac{\gamma^{2}\left(n_{\|}^{2}-1\right) v}{\mu_{\perp}}\left[\overline{\mathbf{A}}_{\perp} \cdot \partial_{r} \mathbf{A}_{\perp}^{\prime}-\left(\overline{\mathbf{A}}_{\perp} \cdot \partial_{\perp}\right) A_{r}^{\prime}\right]\right\}-\left(\overline{\mathbf{A}} \leftrightarrow \mathbf{A}^{\prime}\right)
$$

Below, we evaluate this expression for each type of mode.

\section{TE modes}

a. Stable

Substituting $\mathbf{A}^{(\mathrm{TE})}=\left(0, \partial_{\varphi} \psi / \sin \theta,-\sin \theta \partial_{\theta} \psi\right)$ into Eq. (A1), with $\psi=e^{-i \omega \tau} Y_{\ell m}(\theta, \varphi) f_{\omega \ell}^{(\mathrm{TE})}(r)$, one gets

$$
\begin{aligned}
\left(A^{(\mathrm{TE})}, A^{\prime(\mathrm{TE})}\right)= & \int_{S^{2}} d S\left[\left(\partial_{\theta} \overline{Y_{\ell m}}\right)\left(\partial_{\theta} Y_{\ell^{\prime} m^{\prime}}\right)+\frac{m m^{\prime}}{\sin ^{2} \theta} \overline{Y_{\ell m}} Y_{\ell^{\prime} m^{\prime}}\right] \int_{\mathcal{I}} d \rho e^{i\left(\omega-\omega^{\prime}\right) \tau}\left[\left(\omega+\omega^{\prime}\right) \frac{\varepsilon_{\perp}}{\mu_{\perp}} \overline{f_{\omega \ell}^{(\mathrm{TE})}} f_{\omega^{\prime} \ell^{\prime}}^{(\mathrm{TE})}\right. \\
& \left.+\frac{i \gamma^{2}\left(n_{\|}^{2}-1\right) v}{\mu_{\perp}}\left(\overline{f_{\omega \ell}^{(\mathrm{TE})}} \frac{d}{d \rho} f_{\omega^{\prime} \ell^{\prime}}^{(\mathrm{TE})}-f_{\omega^{\prime} \ell^{\prime}}^{(\mathrm{TE})} \frac{d}{d \rho} \overline{f_{\omega \ell}^{(\mathrm{TE})}}\right)\right],
\end{aligned}
$$

where $S^{2}$ is the unit sphere, recall that $d r / d \rho=\gamma^{2}\left(1-n_{\|}^{2} v^{2}\right) / \mu_{\perp}$, and it is understood that this last integral must be evaluated at $\tau+p(r)=t=$ constant [recall definition of $\tau$ right above Eq. (93)]. It is straightforward to show that the first integral evaluates to $\ell(\ell+1) \delta_{\ell \ell^{\prime}} \delta_{m m^{\prime}}$ provided we normalize $Y_{\ell m}$ according to $\int_{S^{2}} d S \overline{Y_{\ell m}} Y_{\ell^{\prime} m^{\prime}}=\delta_{\ell \ell^{\prime}} \delta_{m m^{\prime}}$. As for the second integral, let us first consider the quantity

$$
W_{\omega \omega^{\prime}}^{(\ell)}:=\frac{1}{\left(\omega-\omega^{\prime}\right)}\left(\overline{f_{\omega \ell}^{(\mathrm{TE})}} \frac{d}{d \rho} f_{\omega^{\prime} \ell}^{(\mathrm{TE})}-f_{\omega^{\prime} \ell}^{(\mathrm{TE})} \frac{d}{d \rho} \overline{f_{\omega \ell}^{(\mathrm{TE})}}\right)
$$


Making use of Eq. (97), $W_{\omega \omega^{\prime}}^{(\ell)}$ clearly satisfies

$$
\frac{d}{d \rho} W_{\omega \omega^{\prime}}^{(\ell)}=\frac{\varepsilon_{\perp}}{\mu_{\perp}}\left(\omega+\omega^{\prime}\right) \overline{f_{\omega \ell}^{(\mathrm{TE})}} f_{\omega^{\prime} \ell}^{(\mathrm{TE})}
$$

Therefore,

$$
\begin{aligned}
\left(A^{(\mathrm{TE})}, A^{\prime(\mathrm{TE})}\right)= & \ell(\ell+1) \delta_{\ell \ell^{\prime}} \delta_{m m^{\prime}} \int_{\mathcal{I}} d \rho e^{i\left(\omega-\omega^{\prime}\right) \tau}\left[\frac{d}{d \rho} W_{\omega \omega^{\prime}}^{(\ell)}-i\left(\omega-\omega^{\prime}\right) \frac{d p}{d \rho} W_{\omega \omega^{\prime}}^{(\ell)}\right] \\
& =\ell(\ell+1) \delta_{\ell \ell^{\prime}} \delta_{m m^{\prime}} \int_{\mathcal{I}} d \rho e^{i\left(\omega-\omega^{\prime}\right)[\tau+p(r)]} \frac{d}{d \rho}\left(e^{-i\left(\omega-\omega^{\prime}\right) p(r)} W_{\omega \omega^{\prime}}^{(\ell)}\right) \\
& =\left.\ell(\ell+1) \delta_{\ell \ell^{\prime}} \delta_{m m^{\prime}} e^{i\left(\omega-\omega^{\prime}\right) t}\left[e^{-i\left(\omega-\omega^{\prime}\right) p(r)} W_{\omega \omega^{\prime}}^{(\ell)}\right]\right|_{\dot{\mathcal{I}}},
\end{aligned}
$$

where we made use that $t=\tau+p(r)$ is kept constant along integration in $r$ (or $\rho$ ) and [..] $\left.\right|_{\dot{\mathcal{I}}}$ indicates that we must calculate the flux of the quantity in square brackets at the boundaries of $\mathcal{I}$. We see that in order to guarantee orthogonality between modes with different $\omega$, without worrying about the specific form of $p(r)$, we must impose boundary conditions at $\dot{\mathcal{I}}$ such that, in Eq. (A3), $\left.W_{\omega \omega^{\prime}}^{(\ell)}\right|_{\dot{\mathcal{I}}}=0$ for $\omega \neq \omega^{\prime}$. Then, referring back to Eq. (A4) and writing

$$
W_{\omega \omega^{\prime}}^{(\ell)}(\rho)=\left(\omega+\omega^{\prime}\right) \int_{\rho_{-}}^{\rho} d \rho^{\prime} \frac{\varepsilon_{\perp}}{\mu_{\perp}} \overline{f_{\omega \ell}^{(\mathrm{TE})}} f_{\omega^{\prime} \ell}^{(\mathrm{TE})},
$$

we finally obtain

$$
\left(A^{(\mathrm{TE})}, A^{\prime(\mathrm{TE})}\right)=2 \omega \ell(\ell+1) \delta_{\ell \ell^{\prime}} \delta_{m m^{\prime}} \int_{\mathcal{I}} d \varrho \overline{f_{\omega \ell}^{(\mathrm{TE})}} f_{\omega^{\prime} \ell}^{(\mathrm{TE})},
$$

which justifies the normalization of the TE modes in Sec. IV. [Notice that the integration variable is $\varrho$, defined through $d r / d \varrho=$ $\left.\gamma^{2}\left(1-n_{\|}^{2} v^{2}\right) / \varepsilon_{\perp} \cdot\right]$

\section{b. Unstable TE modes}

Generic unstable TE modes are given by $\mathbf{A}^{(u \mathrm{TE})}=\left(0, \partial_{\varphi} \psi / \sin \theta,-\sin \theta \partial_{\theta} \psi\right)$ with

$$
\psi=\left(\alpha_{\Omega \ell} e^{\Omega \tau}+\beta_{\Omega \ell} e^{-\Omega \tau}\right) Y_{\ell m}(\theta, \varphi) g_{\Omega \ell}^{(\mathrm{TE})}(r),
$$

where $\alpha_{\Omega \ell}$ and $\beta_{\Omega \ell}$ are complex constants and $g_{\Omega \ell}^{(\mathrm{TE})}(r)$ is a solution of Eq. (97) with $\omega^{2}=-\Omega^{2}(\Omega>0$, without loss of generality) and proper boundary conditions (see below). Sesquilinearity of Eq. (A1) makes it easy to calculate $\left(A^{(u \mathrm{TE})}, A^{\prime(u \mathrm{TE})}\right)$ from Eq. (A5) with the appropriate substitution $\omega \mapsto \mp i \Omega$ and $\omega^{\prime} \mapsto \pm i \Omega^{\prime}$ :

$$
\begin{aligned}
\left(A^{(u \mathrm{TE})}, A^{\prime(u \mathrm{TE})}\right)= & \ell(\ell+1) \delta_{\ell \ell^{\prime}} \delta_{m m^{\prime}}\left[\overline{\alpha_{\Omega \ell}} \alpha_{\Omega^{\prime} \ell} e^{\left(\Omega+\Omega^{\prime}\right) \tau} W_{\Omega \Omega^{\prime}}^{(u \ell)}+\overline{\beta_{\Omega \ell}} \beta_{\Omega^{\prime} \ell} e^{-\left(\Omega+\Omega^{\prime}\right) \tau} W_{-\Omega-\Omega^{\prime}}^{(u \ell)}+\overline{\alpha_{\Omega \ell}} \beta_{\Omega^{\prime} \ell} e^{\left(\Omega-\Omega^{\prime}\right) \tau} W_{\Omega-\Omega^{\prime}}^{(u \ell)}\right. \\
& \left.+\overline{\beta_{\Omega \ell}} \alpha_{\Omega^{\prime} \ell} e^{-\left(\Omega-\Omega^{\prime}\right) \tau} W_{-\Omega \Omega^{\prime}}^{(u \ell)}\right]\left.\right|_{\dot{\mathcal{I}}},
\end{aligned}
$$

where

$$
W_{ \pm \Omega \pm \Omega^{\prime}}^{(u \ell)}:=\frac{i}{\left( \pm \Omega \pm \Omega^{\prime}\right)}\left(\overline{g_{\Omega \ell}^{(\mathrm{TE})}} \frac{d}{d \rho} g_{\Omega^{\prime} \ell}^{(\mathrm{TE})}-g_{\Omega^{\prime} \ell}^{(\mathrm{TE})} \frac{d}{d \rho} \overline{g_{\Omega \ell}^{(\mathrm{TE})}}\right) .
$$

As with the stable case, we must impose boundary conditions on $g_{\Omega \ell}^{(\mathrm{TE})}(r)$ such that

$$
\left.\left(\overline{g_{\Omega \ell}^{(\mathrm{TE})}} \frac{d}{d \rho} g_{\Omega^{\prime} \ell}^{(\mathrm{TE})}-g_{\Omega^{\prime} \ell}^{(\mathrm{TE})} \frac{d}{d \rho} \overline{g_{\Omega \ell}^{(\mathrm{TE})}}\right)\right|_{\dot{\mathcal{I}}}=0,
$$

which implies $\left.W_{\Omega \Omega^{\prime}}^{(u \ell)}\right|_{\dot{I}}=\left.W_{-\Omega-\Omega^{\prime}}^{(u \ell)}\right|_{\dot{I}}=0$ and, for $\Omega \neq \Omega^{\prime},\left.W_{\Omega-\Omega^{\prime}}^{(u \ell)}\right|_{\dot{\mathcal{I}}}=\left.W_{-\Omega \Omega^{\prime}}^{(u \ell)}\right|_{\dot{\mathcal{I}}}=0$. Therefore, using the analogue of Eq. (A6),

$$
W_{ \pm \Omega \pm \Omega^{\prime}}^{(u \ell)}(\rho)=-i\left( \pm \Omega \mp \Omega^{\prime}\right) \int_{\rho_{-}}^{\rho} d \rho^{\prime} \frac{\varepsilon_{\perp}}{\mu_{\perp}} \overline{g_{\Omega \ell}^{(\mathrm{TE})}} g_{\Omega^{\prime} \ell}^{(\mathrm{TE})},
$$

into Eq. (A9), we finally obtain

$$
\left(A^{(u \mathrm{TE})}, A^{\prime(u \mathrm{TE})}\right)=4 \Omega \ell(\ell+1) \delta_{\ell \ell^{\prime}} \delta_{m m^{\prime}} \operatorname{Im}\left(\overline{\alpha_{\Omega \ell}} \beta_{\Omega \ell}\right) \int_{\mathcal{I}} d \varrho \overline{g_{\Omega \ell}^{(\mathrm{TE})}} g_{\Omega^{\prime} \ell}^{(\mathrm{TE})},
$$


where $\operatorname{Im}(z)$ stands for the coefficient of the imaginary part of the complex number $z$. Thus, imposing orthonormality of these modes, for orthonomalized $g_{\Omega \ell}^{(\mathrm{TE})}$ [in the $L^{2}\left(\mathcal{I}, d \varrho\right.$ ) inner product], the general expression for $\alpha_{\Omega \ell}$ and $\beta_{\Omega \ell}$ (up to rephasing, $\alpha_{\Omega \ell} \mapsto e^{i \delta} \alpha_{\Omega \ell}, \beta_{\Omega \ell} \mapsto e^{i \delta} \beta_{\Omega \ell}$, and time resetting, $\alpha_{\Omega \ell} \mapsto e^{\Omega t_{0}} \alpha_{\Omega \ell}, \beta_{\Omega \ell} \mapsto e^{-\Omega t_{0}} \beta_{\Omega \ell}$ ) read as

$$
\begin{aligned}
& \alpha_{\Omega \ell}=\frac{e^{-i \kappa / 2}}{2 \sqrt{\Omega \ell(\ell+1) \sin \kappa}}, \\
& \beta_{\Omega \ell}=\frac{e^{i \kappa / 2}}{2 \sqrt{\Omega \ell(\ell+1) \sin \kappa}},
\end{aligned}
$$

with $0<\kappa<\pi$ being an arbitrary constant.

\section{TM modes}

\section{a. Stable}

Now, substituting $\mathbf{A}^{(\mathrm{TM})}=\left(r^{-2} \varepsilon_{\|}^{-1} \Delta_{S}^{(0)}, \partial_{\theta} \partial_{\varrho}, \partial_{\varphi} \partial_{\varrho}\right) \phi$ into Eq. (A1), with $\phi=e^{-i \omega \tau} Y_{\ell m}(\theta, \varphi) f_{\omega \ell}^{(\mathrm{TM})}(r)$, and evaluating the angular integrals (similarly to the previous TE case), we obtain

$$
\begin{aligned}
\left(A^{(\mathrm{TM})}, A^{\prime(\mathrm{TM})}\right)= & \ell(\ell+1) \delta_{\ell \ell^{\prime}} \delta_{m m^{\prime}} \int_{\mathcal{I}} d \varrho e^{i\left(\omega-\omega^{\prime}\right) \tau}\left\{\left(\omega+\omega^{\prime}\right)\left[\frac{d}{d \varrho} \overline{f_{\omega \ell}^{(\mathrm{TM})}} \frac{d}{d \varrho} f_{\omega^{\prime} \ell}^{(\mathrm{TM})}+\frac{\gamma^{2}\left(1-n_{\|}^{2} v^{2}\right) \ell(\ell+1)}{\varepsilon_{\|} \varepsilon_{\perp} r^{2}} \overline{f_{\omega \ell}^{(\mathrm{TM})}} f_{\omega^{\prime} \ell}^{(\mathrm{TM})}\right]\right. \\
& \left.+\frac{i \gamma^{2}\left(n_{\|}^{2}-1\right) v}{\varepsilon_{\perp}}\left(\omega^{2} \overline{f_{\omega \ell}^{(\mathrm{TM})}} \frac{d}{d \varrho} f_{\omega^{\prime} \ell}^{(\mathrm{TM})}-\omega^{\prime 2} f_{\omega^{\prime} \ell}^{(\mathrm{TM})} \frac{d}{d \varrho} \overline{f_{\omega \ell}^{(\mathrm{TM})}}\right)\right\},
\end{aligned}
$$

where recall that $d r / d \varrho=\gamma^{2}\left(1-n_{\|}^{2} v^{2}\right) / \varepsilon_{\perp}$. The strategy to simplify the expression above is the same applied in the TE case. Define

$$
\mathcal{W}_{\omega \omega^{\prime}}^{(\ell)}:=\frac{1}{\left(\omega-\omega^{\prime}\right)}\left(\omega^{2} \overline{f_{\omega \ell}^{(\mathrm{TM})}} \frac{d}{d \varrho} f_{\omega^{\prime} \ell}^{(\mathrm{TM})}-\omega^{\prime 2} f_{\omega^{\prime} \ell}^{(\mathrm{TM})} \frac{d}{d \varrho} \overline{f_{\omega \ell}^{(\mathrm{TM})}}\right) .
$$

One can easily check, using Eq. (102), that

$$
\frac{d}{d \varrho} \mathcal{W}_{\omega \omega^{\prime}}^{(\ell)}=\left(\omega+\omega^{\prime}\right)\left[\frac{d}{d \varrho} \overline{f_{\omega \ell}^{(\mathrm{TM})}} \frac{d}{d \varrho} f_{\omega^{\prime} \ell}^{(\mathrm{TM})}+\frac{\gamma^{2}\left(1-n_{\|}^{2} v^{2}\right) \ell(\ell+1)}{\varepsilon_{\|} \varepsilon_{\perp} r^{2}} \overline{f_{\omega \ell}^{(\mathrm{TM})}} f_{\omega^{\prime} \ell}^{(\mathrm{TM})}\right]
$$

Therefore, we can put Eq. (A14) in the same form as Eq. (A5), with $W \mapsto \mathcal{W}$ and $\rho \mapsto \varrho$. Now, orthogonality of the modes demand that $f_{\omega \ell}^{(\mathrm{TM})}$ satisfy either Dirichlet or Neumann boundary conditions at $\dot{\mathcal{I}}$, which leads to

$$
\left(A^{(\mathrm{TM})}, A^{\prime(\mathrm{TM})}\right)=\left.\ell(\ell+1) \delta_{\ell \ell^{\prime}} \delta_{m m^{\prime}}\left[\mathcal{W}_{\omega \omega^{\prime}}^{(\ell)}\right]\right|_{\dot{\mathcal{I}}} .
$$

In order to simplify even further the expression above, note that using again Eq. (102) in Eq. (A16), we can write

$$
\left.\frac{d}{d \varrho} \mathcal{W}_{\omega \omega^{\prime}}^{(\ell)}=\frac{\left(\omega+\omega^{\prime}\right)}{2}\left[\frac{d^{2}}{d \varrho^{2}}+\frac{\mu_{\perp}}{\varepsilon_{\perp}}\left(\omega^{2}+\omega^{\prime 2}\right)\right] \overline{\left(f_{\omega \ell}^{(\mathrm{TM})}\right.} f_{\omega^{\prime} \ell}^{(\mathrm{TM})}\right),
$$

whose integration on $\mathcal{I}$ gives us $\left[\mathcal{W}_{\omega \omega^{\prime}}^{(\ell)}\right]_{\dot{\mathcal{I}}}$, which substituted into Eq. (A17) finally leads to

$$
\left(A^{(\mathrm{TM})}, A^{\prime(\mathrm{TM})}\right)=2 \omega^{3} \ell(\ell+1) \delta_{\ell \ell^{\prime}} \delta_{m m^{\prime}} \int_{\mathcal{I}} d \rho \overline{f_{\omega \ell}^{(\mathrm{TM})}} f_{\omega^{\prime} \ell}^{(\mathrm{TM})} .
$$

(Notice that the integration variable is $\rho$.)

\section{b. Unstable}

Generic unstable TM modes are given by $\mathbf{A}^{(u \mathrm{TM})}=\left(r^{-2} \varepsilon_{\|}^{-1} \Delta_{S}^{(0)}, \partial_{\theta} \partial_{\varrho}, \partial_{\varphi} \partial_{\varrho}\right) \phi$ with

$$
\phi=\left(\alpha_{\Omega \ell} e^{\Omega \tau}+\beta_{\Omega \ell} e^{-\Omega \tau}\right) Y_{\ell m}(\theta, \varphi) g_{\Omega \ell}^{(\mathrm{TM})}(r),
$$

where $\alpha_{\Omega \ell}$ and $\beta_{\Omega \ell}$ are complex constants and $g_{\Omega \ell}^{(\mathrm{TM})}(r)$ is a solution of Eq. (102) with $\omega^{2}=-\Omega^{2}(\Omega>0$, without loss of generality) satisfying Dirichlet or Neumann boundary conditions. Once more, sesquilinearity of Eq. (A1) makes it easy to calculate $\left(A^{(u \mathrm{TM})}, A^{\prime(u \mathrm{TM})}\right)$ from Eq. (A17) with the substitution $\omega \mapsto \mp i \Omega$ and $\omega^{\prime} \mapsto \pm i \Omega^{\prime}$ :

$$
\left(A^{(u \mathrm{TM})}, A^{\prime(u \mathrm{TM})}\right)=\left.\ell(\ell+1) \delta_{\ell \ell^{\prime}} \delta_{m m^{\prime}}\left[\overline{\alpha_{\Omega \ell}} \beta_{\Omega^{\prime} \ell} \mathcal{W}_{\Omega-\Omega^{\prime}}^{(u \ell)}+\overline{\beta_{\Omega \ell}} \alpha_{\Omega^{\prime} \ell} \mathcal{W}_{-\Omega \Omega^{\prime}}^{(u \ell)}\right]\right|_{\dot{\mathcal{I}}},
$$


where

$$
\begin{aligned}
\mathcal{W}_{ \pm \Omega \pm \Omega^{\prime}}^{(u \ell)} & :=\frac{-i}{\left( \pm \Omega \pm \Omega^{\prime}\right)}\left(\Omega^{2} \overline{g_{\Omega \ell}^{(\mathrm{TM})}} \frac{d}{d \rho} g_{\Omega^{\prime} \ell}^{(\mathrm{TM})}-\Omega^{\prime 2} g_{\Omega^{\prime} \ell}^{(\mathrm{TM})} \frac{d}{d \rho} \overline{g_{\Omega \ell}^{(\mathrm{TM})}}\right) \\
& =\frac{i\left( \pm \Omega \mp \Omega^{\prime}\right)}{2}\left[-\frac{d}{d \varrho}\left(\overline{g_{\Omega \ell}} \frac{(\mathrm{TM})}{g_{\Omega^{\prime} \ell}^{(\mathrm{TM})}}\right)+\left(\Omega^{2}+\Omega^{\prime 2}\right) \int_{\varrho_{-}}^{\varrho} d \varrho^{\prime} \frac{\mu_{\perp}}{\varepsilon_{\perp}} \overline{g_{\Omega \ell}^{(\mathrm{TM})}} g_{\Omega^{\prime} \ell}^{(\mathrm{TM})}\right] .
\end{aligned}
$$

In the last passage of the expression above we used the analogue of Eq. (A18):

$$
\left(A^{(u \mathrm{TM})}, A^{\prime(u \mathrm{TM})}\right)=-4 \Omega^{3} \ell(\ell+1) \delta_{\ell \ell^{\prime}} \delta_{m m^{\prime}} \operatorname{Im}\left(\overline{\alpha_{\Omega \ell}} \beta_{\Omega \ell}\right) \int_{\mathcal{I}} d \rho \overline{g_{\Omega \ell}^{(\mathrm{TM})}} g_{\Omega^{\prime} \ell}^{(\mathrm{TM})} .
$$

Imposing orthonormality of these modes, for orthonormalized $g_{\Omega \ell}^{(\mathrm{TM})}$ [in the $L^{2}(\mathcal{I}, d \rho)$ inner product], the general expression for $\alpha_{\Omega \ell}$ and $\beta_{\Omega \ell}$ (again, up to rephasing and time resetting) can be expressed as

$$
\begin{aligned}
& \alpha_{\Omega \ell}=\frac{e^{i \kappa / 2}}{2 \sqrt{\Omega^{3} \ell(\ell+1) \sin \kappa}}, \\
& \beta_{\Omega \ell}=\frac{e^{-i \kappa / 2}}{2 \sqrt{\Omega^{3} \ell(\ell+1) \sin \kappa}},
\end{aligned}
$$

with $0<\kappa<\pi$.

[1] W. Gordon, Zur Lichtfortpflanzung nach der Relativitätstheorie, Ann. Phys. (Leipzig) 72, 421 (1923).

[2] W. G. Unruh, Experimental Black-Hole Evaporation? Phys. Rev. Lett. 46, 1351 (1981).

[3] M. Visser, Acoustic black holes: Horizons, ergospheres and Hawking radiation, Classical Quantum Gravity 15, 1767 (1998).

[4] C. H. G. Bessa, V. A. De Lorenci, L. H. Ford, and C. C. H. Ribeiro, Model for lightcone fluctuations due to stress tensor fluctuations, Phys. Rev. D 93, 064067 (2016).

[5] P. Jain, S. Weinfurtner, M. Visser, and C. W. Gardiner, Analog model of a Friedmann-Robertson-Walker universe in BoseEinstein condensates: Application of the classical field method, Phys. Rev. A 76, 033616 (2007).

[6] S. Weinfurtner, E. W. Tedford, M. C. J. Penrice, W. G. Unruh, and G. A. Lawrence, Measurement of Stimulated Hawking Emission in an Analogue System, Phys. Rev. Lett. 106, 021302 (2011).

[7] W. G. Unruh, Has Hawking radiation been measured? Found. Phys. 44, 532 (2014).

[8] J. R. M. de Nova, K. Golubkov, V. I. Kolobov, and J. Steinhauer, Observation of thermal Hawking radiation and its temperature in an analog black hole, Nature (London) 569, 688 (2019).

[9] W. C. C. Lima and D. A. T. Vanzella, Gravity-Induced Vacuum Dominance, Phys. Rev. Lett. 104, 161102 (2010).

[10] W. C. C. Lima, G. E. A. Matsas, and D. A. T. Vanzella, Awaking the Vacuum in Relativistic Stars, Phys. Rev. Lett. 105, 151102 (2010).

[11] W. C. C. Lima, R. F. P. Mendes, G. E. A. Matsas, and D. A. T. Vanzella, Awaking the vacuum with spheroidal shells, Phys. Rev. D 87, 104039 (2013).

[12] R. F. P. Mendes, G. E. A. Matsas, and D. A. T. Vanzella, Instability of nonminimally coupled scalar fields in the spacetime of slowly rotating compact objects, Phys. Rev. D 90, 044053 (2014).
[13] A. G. S. Landulfo, W. C. C. Lima, G. E. A. Matsas, and D. A. T. Vanzella, From quantum to classical instability in relativistic stars, Phys. Rev. D 91, 024011 (2015).

[14] P. Pani, V. Cardoso, E. Berti, J. Read, and M. Salgado, Vacuum revealed: The final state of vacuum instabilities in compact stars, Phys. Rev. D 83, 081501(R) (2011).

[15] T. Damour and G. Esposito-Farèse, Nonperturbative StrongField Effects in Tensor-Scalar Theories of Gravitation, Phys. Rev. Lett. 70, 2220 (1993).

[16] T. Damour and G. Esposito-Farèse, Tensor-scalar gravity and binary-pulsar experiments, Phys. Rev. D 54, 1474 (1996).

[17] E. Berti, Astrophysical black holes as natural laboratories for fundamental physics and strong-field gravity, Braz. J. Phys. 43, 341 (2013).

[18] L. Annulli, V. Cardoso, and L. Gualtieri, Electromagnetism and hidden vector fields in modified gravity theories: Spontaneous and induced vectorization, Phys. Rev. D 99, 044038 (2019).

[19] E. S. Weibel, Spontaneously Growing Transverse Waves in a Plasma Due to an Anisotropic Velocity Distribution, Phys. Rev. Lett. 2, 83 (1959).

[20] N. Rubab, A. C.-L. Chian, and V. Jatenco-Pereira, On the ordinary mode Weibel instability in space plasmas: A comparison of three-particle distributions, J. Geophys. Res. Space Phys. 121, 1874 (2016).

[21] T. A. Morgado and M. G. Silveirinha, Negative Landau Damping in Bilayer Graphene, Phys. Rev. Lett. 119, 133901 (2017).

[22] B. Nistad and J. Skaar, Causality and electromagnetic properties of active media, Phys. Rev. E 78, 036603 (2008).

[23] R. Matloob, R. Loudon, S. M. Barnett, and J. Jeffers, Electromagnetic field quantization in absorbing dielectrics, Phys. Rev. A 52, 4823 (1995).

[24] R. Matloob, R. Loudon, M. Artoni, S. M. Barnett, and J. Jeffers, Electromagnetic field quantization in amplifying dielectrics, Phys. Rev. A 55, 1623 (1997). 
[25] C. Raabe, S. Scheel, and D.-G. Welsch, Unified approach to QED in arbitrary linear media, Phys. Rev. A 75, 053813 (2007).

[26] B. Huttner and S. M. Barnett, Quantization of the electromagnetic field in dielectrics, Phys. Rev. A 46, 4306 (1992).

[27] C. Raabe and D.-G. Welsch, QED in arbitrary linear media: Amplifying media, Eur. Phys. J. Special Topics 160, 371 (2008).

[28] A. G. S. Landulfo, W. C. C. Lima, G. E. A. Matsas, and D. A. T. Vanzella, Particle creation due to tachyonic instability in relativistic stars, Phys. Rev. D 86, 104025 (2012).

[29] R. M. Wald, General Relativity (University of Chicago Press, Chicago, 1984).

[30] By "spatial" here we mean that the contraction of any index of these tensors with the medium's four-velocity $v^{a}$ vanishes.

[31] U. Leonhardt and P. Piwnicki, Optics of nonuniformly moving media, Phys. Rev. A 60, 4301 (1999).

[32] U. Leonhardt and P. Piwnicki, Relativistic Effects of Light in Moving Media with Extremely Low Group Velocity, Phys. Rev. Lett. 84, 822 (2000).

[33] I. T. Drummond and S. J. Hathrell, QED vacuum polarization in a background gravitational field and its effect on the velocity of photons, Phys. Rev. D 22, 343 (1980).

[34] A. B. Balakin, V. V. Bochkarev, and J. P. S. Lemos, Nonminimal coupling for the gravitational and electromagnetic fields: Black hole solutions and solitons, Phys. Rev. D 77, 084013 (2008).

[35] A. B. Balakin and J. P. S. Lemos, Non-minimal coupling for the gravitational and electromagnetic fields: a general system of equations, Classical Quantum Gravity 22, 1867 (2005).

[36] R. J. Glauber and M. Lewenstein, Quantum optics of dielectric media, Phys. Rev. A 43, 467 (1991).

[37] A. M. Contreras Reyes and C. Eberlein, Completeness of evanescent modes in layered dielectrics, Phys. Rev. A 79, 043834 (2009).
[38] C. K. Carniglia and L. Mandel, Quantization of evanescent electromagnetic waves, Phys. Rev. D 3, 280 (1971).

[39] D. R. Smith, W. J. Padilla, D. C. Vier, S. C. Nemat-Nasser, and S. Schultz, Composite Medium with Simultaneously Negative Permeability and Permittivity, Phys. Rev. Lett. 84, 4184 (2000).

[40] R. A. Shelby, D. R. Smith, and S. Schultz, Experimental verification of a negative index of refraction, Science 292, 77 (2001).

[41] W. J. Padilla, D. N. Basov, and D. R. Smith, Negative refractive index metamaterials, Mater. Today 9, 28 (2006).

[42] A. Poddubny, I. Iorsh, P. Belov, and Y. Kivshar, Hyperbolic metamaterials, Nat. Photonics 7, 948 (2013).

[43] V. Caligiuri, R. Dhama, K. Sreekanth, G. Strangi, and A. De Luca, Dielectric singularity in hyperbolic metamaterials: the inversion point of coexisting anisotropies, Sci. Rep. 6, 20002 (2016).

[44] C. C. H. Ribeiro and D. A. T. Vanzella (unpublished).

[45] H. Minkowski, Die Grundgleichungen für die elektromagnetischen Vorgänge in bewegten Körpern, Math. Ann. 68, 472 (1910).

[46] A. V. Andreev, I. L. Aleiner, and A. J. Millis, Dynamical Symmetry Breaking as the Origin of the Zero-dc-Resistance State in an ac-Driven System, Phys. Rev. Lett. 91, 056803 (2003).

[47] R. Mani, J. H. Smet, K. von Klitzing, V. Narayanamurti, W. B. Johnson, and V. Umansky, Zero-resistance states induced by electromagnetic-wave excitation in GaAs/AlGaAs heterostructures, Nature (London) 420, 646 (2002).

[48] M. A. Zudov, R. R. Du, L. N. Pfeiffer, and K. W. West, Evidence for a New Dissipationless Effect in 2D Electronic Transport, Phys. Rev. Lett. 90, 046807 (2003).

[49] J. Inarrea and G. Platero, Theoretical Approach to MicrowaveRadiation-Induced Zero-Resistance States in 2D Electron Systems, Phys. Rev. Lett. 94, 016806 (2005); 111, 229903(E) (2013). 